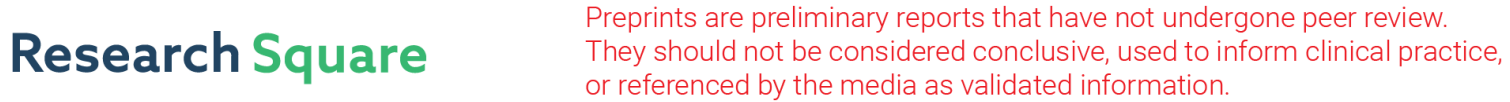 \\ Shift Quality Optimization of Clutchless Automatic Mechanical Transmission Based on a Novel Synchronizer
}

Lipeng Zhang ( $\square$ evzlp@163.com )

Yanshan University https://orcid.org/0000-0001-5204-8610

Yun-ao Peng

Yanshan University

Haojie Yang

Yanshan University

Original Article

Keywords: Electric vehicle, Clutchless automatic mechanical transmission, Shift quality, Mechanical characteristics, Inertial lock-ring synchronizer, Pressure- controllable friction ring synchronize

Posted Date: May 5th, 2021

DOl: https://doi.org/10.21203/rs.3.rs-473977/v1

License: (c) (i) This work is licensed under a Creative Commons Attribution 4.0 International License. Read Full License 


\section{Title page}

\section{Shift Quality Optimization of Clutchless Automatic Mechanical Transmission Based on a Novel Synchronizer}

Li-Peng Zhang, born in 1979, is currently a professor at Yanshan University, China. He received his PhD degree from Beijing Institute of Technology, China, in 2011. His research interests include intelligent vehicle dynamics and control, new energy vehicle compound transmission, driver awareness and driving assistance.

Tel: +86-18533537646; E-mail: evzlp@ysu.edu.cn

Yun-Ao Peng, born in 1996, is currently a master candidate at Hebei Key Laboratory of Special Delivery Equipment, Yanshan University, China.

E-mail: 2667940666@qq.com

Hao-Jie Yang, born in 1998, is currently a master candidate at Hebei Key Laboratory of Special Delivery Equipment, Yanshan University, China.

E-mail: 798056973@qq.com

Corresponding author: Li-Peng Zhang E-mail: evzlp@ysu.edu.cn 


\title{
Shift Quality Optimization of Clutchless Automatic Mechanical Transmission Based on a Novel Synchronizer
}

\author{
ZHANG LiPeng $^{1,2} \cdot$ PENG YunAo $^{1} \cdot$ Yang HaoJie $^{1}$
}

Received June xx, 201x; revised February xx, 201x; accepted March xx, 201x

(C) Chinese Mechanical Engineering Society and Springer-Verlag Berlin Heidelberg 2017

\begin{abstract}
The synchronizer is a key component of the clutchless automatic mechanical transmission (AMT) for electric vehicles, but the inertial lock-ring synchronizer (ILRS) commonly used there is not very suitable for pure electric vehicles. To realize the rapid and smooth shifting with little impact, a new synchronizer named pressure-controllable friction ring synchronizer (PCFRS) is researched. Initially, the mechanical characteristics of the new synchronizer are analyzed, and the advantages over the ILRS are reflected. Furthermore, the dynamic transmission formulation based on the working mechanism of the PCFRS is established. Finally, the shift effects of the two kinds of synchronizers are simulated and compared. The research shows that the PCFRS can meet the main shifting evaluation index of an AMT without complex control methods, as well as it takes only $0.2406 \mathrm{~s}$ to finish the comfortable shifting-no speed difference shifting. The shifting quality of PCFRS is better than that of the ILRS. It lays the foundation for the use of the new synchronizer for the clutchless AMTs of electric vehicles.
\end{abstract}

Keywords: Electric vehicle, Clutchless automatic mechanical transmission, Shift quality, Mechanical characteristics, Inertial lock-ring synchronizer, Pressurecontrollable friction ring synchronize,

\section{Introduction}

Pure electric vehicles, as the representative of new energy vehicles, is the important researching objects in the 1

\footnotetext{
$\bowtie$ Li-Peng Zhang evzlp@ysu.edu.cn

${ }^{1}$ Hebei Key Laboratory of Special Delivery Equipment, Yanshan University, Qinhuangdao 066004, China

${ }^{2}$ School of Vehicle and Energy, Yanshan University, Qinhuangdao 066004
}

automotive industry in response to the energy crisis and environmental pollution [1]. The automatic mechanical transmission (AMT) can balance power and economy, as well as improve the lifespan of the motor and the controller unit [2], so it is in vogue to use an AMT in the transmission systems of pure electric vehicles. For the electric motor of pure electric vehicles, the driving torque and speed can be controlled more easily, the motor can reduce the need for clutches with the active speed regulation, and the clutchless AMT is increasingly used $[3,4]$. The shifting quality of clutchless AMT directly affects the dynamic performance, economy and driving comfort of an electric vehicle [5]. Thus, a synchronizer with better characteristics is badly need for the clutchless AMT because the synchronizer is used to achieve the speed synchronization between the sleeve and the gear ring $[6,7]$. To be specific, it can reduce the shifting impact and noise. In addition, it can make the shifting process steady and extend the service lifespan of AMTs [8]. As the core component, its performance has an important influence on the shifting quality. So the research on synchronizer is significance.

Different shifting control strategies based on the working mechanism of ILRS are proposed to improve the shift quality. Shifting force is a key factor affecting shifting quality. Wang et al. adopted a position and force switching control scheme to avoid the problem that the shifting force produces a large impact [9]. For the lock position, as the shift occurs, the friction coefficient between the synchro ring and the friction cone ring will decrease and that will also result in the shift failure [10]. Besides, the rapid adjustment of the drive motor speed and the rapid teeth feeding of the sleeve may cause the synchronizer to fail to lock teeth. Qin et al. adopted a gear shift control strategy based on parallel coordinated control to realize reliable, smooth and fast gear shifting [11]. In addition, the input shaft is directly connected with the drive motor, resulting in a greater inertia of the input shaft, therefore a greater impact when the gears are engaged. For 
the above questions, Lin et al. designed an inverse system and an active disturbance rejection controller to reduce the impact and synchronization time [12]. Zhang et al. proposed a stage-by-phase multivariable combination controller based on the control of position, velocity and force of the actuators, which reduces the impact of mode switching and improves the dynamics performance of electric vehicles [13]. Zhang et al. proposed a synchronizer shift trajectory tracking controller [14]. However, due to the very short shifting time, the control algorithms may be too complex to complete system identification and parameters correction. Besides, due to the randomness of shifting and the wear of mechanical parts, the reliability of this strategy needs to be improved.

The structural parameters of the synchronizer have various effects on the synchronization performance, locking performance, shift quality and service lifespan [15]. Different parts of synchronization also have different degree of influence [16,17]. Wang et al. optimized the parameters of synchronizer to effectively shorten the synchronization time and increase the lifespan of the synchronizer [18]. The researchers have effectively improved the shift quality of the AMT using the inertial lock-ring synchronizers (ILRSs). However, in combination with the characteristics of the ILRS and the electric motor, the following problems still exist: First of all, the sleeve cannot be moved before completing synchronization. Secondly, there is a break-through load when the sleeve breaks away from the slider [19], which can't be predicted and difficult to control when it moves. Thirdly, the ILRS will cause two large impact during the gear shift. At last, combined with the shifting process of the ILRS, the synchro ring will not work after the synchronization phase, and the speed difference will be generated in the final turn-teeth phase, and then it will cause a huge impact [20].

With the application of electric motors in the new energy vehicles, the clutchless mechanical direct-connection motor-transmission systems are increasingly used in the electric vehicles $[3,4]$. The research on the direct-connection motor-transmission system mainly focuses on the application of various motor control algorithms to achieve the speed synchronization of the sleeve and the gear ring [6,7], and the feasibility of using adapter sleeves to shift gears. Zeng et al. designed a gear shift control strategy that combines active rotation speed synchronization and rotation angle active synchronization to achieve precise synchronization between the sleeve and the combined gear ring [21]. Tian et al. introduced the "zero speed difference" and "zero rotation angle difference" dual target tracking control algorithm, which solves the problem of frequent switching between the driving and braking of the drive motor [22]. The researches have made great progress in canceling the synchronizer and directly using the sleeve to shift gears, which is a reference for the development of clutchless AMT. However, the direct shifting method using the sleeve requires high control accuracy and equipment conditions, and the control algorithm requires high real-time performance of the system, which makes it difficult to use the sleeve for shifting.

Regarding the electromechanical coupling of gear shifting in pure electric vehicles, some scholars have studied novel synchronization mechanisms to replace traditional synchronizers to achieve gear shifting. The British innovation company Zeroshift has developed a new system that uses the Zeroshift meshing ring mechanism to replace the synchronizer, and uses two sliders to achieve shifting without power interruption [23]. However, two sets of shift forks are needed to realize the shift, which makes the shift actuator complicated and requires more complicated shift control. Mo et al. proposed a new type of synchronizer called harpoon-shift, which uses a torsion spring to replace the friction cone ring of the ILRS to eliminate the speed difference. This mechanism overcomes the shortcomings of friction synchronous shifting [24,25]. However, the use of torsion springs greatly limits the range of the shift speed difference. If the speed difference between the sleeve and the meshing gear ring is too large, the spring will be directly compressed to the maximum, resulting in greater impact; if the speed difference is too small, there may be a speed difference in the gear advancement process.

In summary, the clutchless AMT of pure electric vehicles using traditional ILRSs is not very suitable; the way of directly canceling the synchronizer and adopting the method of sleeve shifting is relatively difficult and requires high-precision sensors, there are many limiting factors. Therefore, a better gear shifting scheme is to use a suitable synchronizer for its shifting with easy-to-achieve shift control. However, there is currently no shift mechanism that is very suitable. In response to the above problems, a pressure-controllable friction ring synchronizer(PCFRS) is proposed in the paper. This novel synchronizer realizes synchronization through compression springs and does not require lock teeth of gear during synchronization. The sleeve quickly reaches the designated position, and there is no problem of inability to synchronize and teeth of gear due to lock failure. It is also possible to advance the gear when the speed difference reaches the allowable gear advancement range. There is no slider mechanism, that is, it is not necessary for the sleeve to apply the overtaking force that breaks off the constraint of the slider. In the gear shifting stage, the generated speed difference can be eliminated synchronously, so as to achieve no speed difference shift. This paper will analyze the shift mechanism and mechanical characteristics of the PCFRS. A control method is adopted to realize the shift. The shift 
characteristics of the synchronizer in the shift process are analyzed and whether the evaluation indicators meet the allowable value is judged. The evaluation indicators include the specific sliding work, the product of the ratio of the pressure on the friction cone to the contact area and the relative sliding speed ( $\mathrm{PV}$ value). At last, the commonly used ILRS is compared to highlight the performance of the new synchronizer.

The structure of the paper is as follows. Section 2 introduces the shift strategy. Section 3 establishes the dynamics model of the meshing. Section 4 simulates gear shifting and analyzes the simulation results. Section 5 provides the shift performance simulation comparison results between the ILRS and the PCFRS. Section 6 draws the conclusion, summarizes and looks forward to this paper.

\section{Working mechanism Analysis of the new synchronizer}

The structure of PCFRS is shown in Fig. 1, which includes hub, sleeve, wave spring, synchro ring, friction cone ring. The synchro ring and the sleeve are used to eliminate the speed difference and engage the target gear. The hub is connected to the input shaft for restraining and transmitting the movement of the sleeve and the synchro ring. The shifting force is transmitted by the spring between the sleeve and the synchro ring. After the synchronization is completed, the sleeve directly compresses the spring into the teeth to realize the turn-teeth and complete the shift. If the rotational speed of the drive motor is directly adjusted to the speed range of the gear advancement, the sleeve is directly controlled to complete the shift. If the rotational speed does not reach the gear-advancing speed range, the sleeve will move a certain distance and wait until the gear-advancing speed range is synchronously reached before completing the gear shift to ensure the reliability of gear shifting.

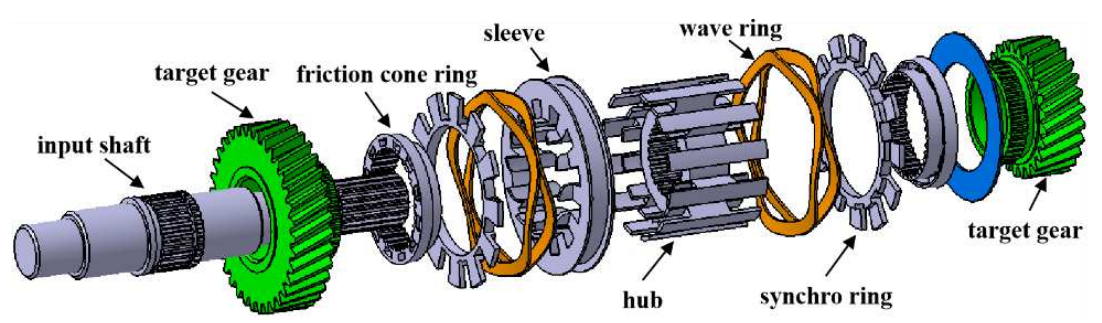

Fig.1. The structure and assembly relationship of PCFRS

The functions of the important parts of the PCFRS are as follows:

The hub is fixed on the input shaft, which connected to the drive motor, constrains the synchro ring and the sleeve to rotate at the same speed, so that the input shaft completes the same speed with the target gear and guides the sleeve into the gear shifting gear to complete the shift.

The sleeve is moved axially on the hub, connects the target gear and the input shaft through the hub to achieve drive. In the process of shifting, the sleeve teeth with a specific teeth profile contact the internal teeth of the friction cone ring, and the sleeve and the friction cone ring rotate relative to each other under the action of the teeth surface contact force, and the sleeve continues to move and then completes the shift.

The synchro ring is the friction element. It frictions with the friction cone ring fixed on the target gear to realize the synchronization of the input shaft and the target gear speed.

The two ends of the wave spring contact the sleeve and the synchro ring respectively. When shifting, the sleeve is moved axially and pushes the wave spring to contact the synchro ring. The synchro ring is contacted with the friction cone ring under the action of spring compression. The spring is further compressed to complete the gear shift.
In the process of shifting, keeps the synchro ring in contact with the friction cone ring. The wave spring strengthens the synchronization effect of the synchro ring on the speed difference generated by the gear shifting, and promotes the completion of the gear shift.

The friction cone ring is fixed on the target gear, the outer side friction with the synchro ring to synchronize the speed, the end surface is opened with internal teeth holes to guide the sleeve teeth to complete the gear shift.

Compared with the ILRS, the PCFRS has the following advantages:

(1) There is no pre-synchronization and no need to squeeze the slider with excess force during the shifting process, which makes the shifting process control more stable and reliable.

(2) There is no lock teeth and turn-ring phase, and there is no need to worry about the inability to synchronize the speed due to the failure of the lock teeth of the synchro ring, which saves shift time, does not need to produce two shift impacts and makes the synchronizer work more reliable.

(3) The sleeve can move before the speed difference is completely eliminated in the synchronization phase, and it can be set to move when the speed difference reaches the allowable range, which make shifting more efficient. 
(4) The working characteristics are in line with the speed regulation characteristics of the motor, and the work is flexible and effective. According to different speed differences, it can adopt direct shifting or shifting after synchronization to the corresponding speed difference, which is more suitable for the shifting of pure electric vehicle transmissions.

(5) Synchronous friction torque always exists during the shifting process, so that the speed difference generated after turn-teeth is quickly reduced, and the impact generated is also smaller, and impact-free shifting can be realized, making shifting easier to control.

(6) There are fewer components than ILRS, there is no sliding block mechanism, and the work is stable and there is no sudden shifting force. The PCFRS has a simpler structure and fewer teeth. Therefore, the reliability is higher, all the components are easy to process, and the cost is lower.

The limitation of the design is that the friction is still needed to achieve synchronous speed and then to shift. The friction will bring losses and affect the lifespan of friction elements. However, according to relevant literature studies, pure electric vehicles with the characteristics of motors are suitable for two-speed transmissions [26-28].

At low speeds or when sufficient power is required, the first gear of the transmission is used for driving. When high-speed driving does not require large power, the second gear of the transmission is used for driving. It can be seen that the low-gear transmission of pure electric vehicles does not shift frequently, thus reducing the drawbacks of friction loss in shifting. In order to highlight the mechanical characteristics of the PCFRS, this paper will compare the shift characteristics of the PCFRS and the ILRS. In order to verify that the PCFRS adopts wave springs to meet the working requirements during the entire shifting process, this paper will verify the slipping work and working conditions of each shift. In addition, the friction always exists during the shifting process, and the shifting actuator will always bear the pressure brought by the wave spring, but this pressure is not very large. The problem can be solved by adopting a self-locking actuator, and the researched synchronizer is equipped with a worm gear shift actuator. According to the self-locking characteristics of the worm gear, the shift function of the synchronizer will be well realized. The simple shifting control strategy of the pressure controllable friction ring synchronizer is as follows:

(1) The sleeve moves a certain distance, and then the wave spring is compressed to make the synchronization ring contact the friction cone ring for synchronization;

(2) When the speed difference reaches the required range, the sleeve will continue to move at a certain speed;

(3) After the sleeve contacts the internal teeth of the friction cone ring, the contact force of the teeth surface causes a certain speed difference, and then the latter two separate;

(4) The sleeve continues to move at a certain speed, and the friction element eliminates the speed difference at a faster speed while the sleeve moves, and finally realizes the zero speed difference shift.

\section{Phased dynamics modeling of the synchronization process}

The model of the transmission system with a PCFRS is shown in Fig. 2, where s represents the sleeve, $r$ represents the synchro ring, and g represents the target gear. $I_{l}$ is the moment of inertia of the motor. $I_{g}$ is the moment of inertia of the target gear. $I_{x}, I_{e}, I_{f}$ are the moment of inertia of the meshing gear, $I_{v}$ is the moment of inertia of the wheel. $T_{l}$, $T_{v}$ are drive torque of the motor and wheel torque. $c_{i}$ is the damping coefficient of the corresponding component, where $i=0,1,2,3,4 . k_{i}$ is the stiffness coefficient of the corresponding component, where $i=0,2$. The PCFRS is mounted on the input shaft. The motor inputs torque to the input shaft. After the gear is engaged, the target gear on the input shaft transmits the torque to the intermediate shaft gear, and the torque is transmitted to the wheels through the gear meshing of the intermediate shaft and the output shaft. Therefore, this section will combine the transmission system to analyze the dynamics characteristics of the PCFRS.

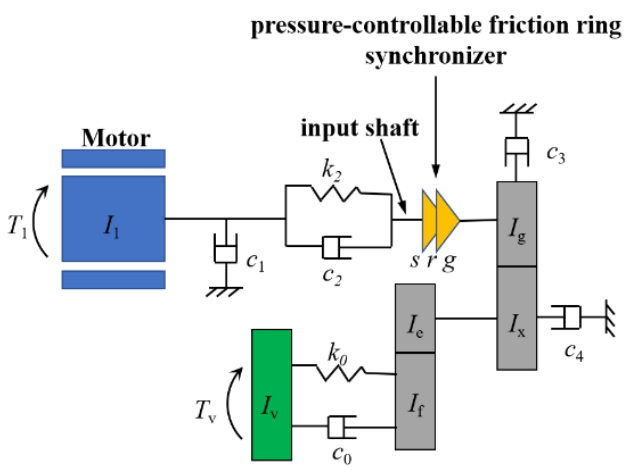

Fig.2. The transmission system with PCFRS

In order to facilitate the analysis of the shifting process of the PCFRS, it is simplified into four main components, the sleeve, the wave spring, the synchro ring and the friction cone ring, as shown in Fig. 4. The friction cone ring is fixed on the target gear. After the synchro ring contacts the friction cone ring, the synchronization phase is completed by friction. The sleeve teeth contact with the internal teeth of the friction cone ring to turn the teeth and then complete shift, so friction cone ring is regarded as a part of the target gear. The whole process of the shifting of the sleeve is divided into multiple stages. In fact, the process of synchronously eliminating the speed difference 
and the timing of shifting are both random, so that the relative position of the sleeve and the target gear during the shifting process is also random when meshing. Therefore, in combination with the randomness of shifting, according to the different conditions of the speed difference and the position of the sleeve, this section selects the most representative stages that can cover all situations. Combined with the dynamics model establishment method proposed by Zhang et al [29], it is expressed as follows.

In order to express the dynamics relationship of the gear meshing process more clearly, this paper adopts the gear contact model [30], as shown in Fig. 3, where $R_{a}$ and $R_{b}$ are the basic radius of the gear, $\theta_{a}$ and $\theta_{b}$ are the torsional freedom of the driving gear and the driven gear, $c_{y 1}, c_{y 2}, k_{y 1}$ and $k_{y 2}$ are the supporting damping coefficient and supporting stiffness of the driving gear and the driven gear, respectively. $c_{m}$ and $k_{m}$ are the damping coefficient and stiffness of the driving gear and driven gear meshing. $I_{a}, I_{b}$ are the moment of inertia of the meshing gear. $e$ is the static transmission error of the gear pair.

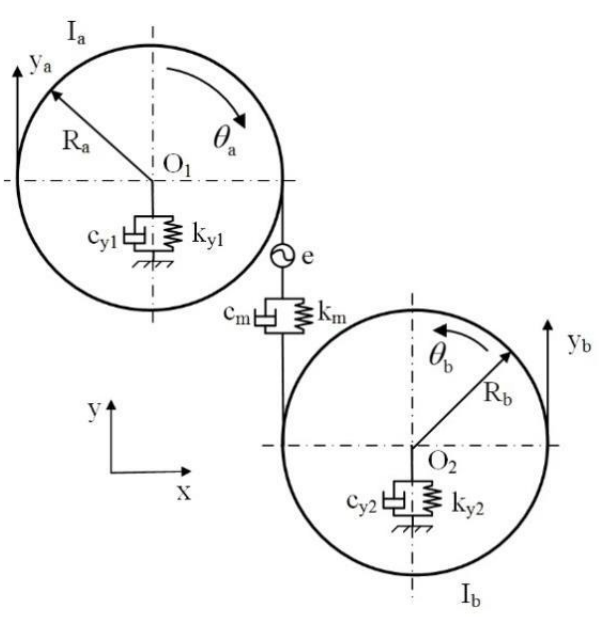

Fig.3. Non-linear dynamics model of gear meshing

The relative displacement of the driving gear and the driven gear due to torsion in the normal coordinate system is

$$
y_{0}=R_{a} \theta_{a}-R_{b} \theta_{b}
$$

Stage 1: The sleeve moves to make the synchro ring contact the friction cone ring.

The state of PCFRS in the first stage is shown in Fig. 4. At this stage, the sleeve is in the middle position of the synchronizer, and the wave spring is in the free length state. The sleeve moves axially toward the target gear under the guiding action of the hub, pushing the wave spring to contact the synchro ring. The sleeve that continues to move causes the wave spring to be compressed, and the spring force pushes the synchro ring to move axially toward the target gear under the guidance of the hub and the synchro ring quickly contact the friction cone surface on the outside of the friction cone ring. When the sleeve continues to move and reaches the set position, the wave spring gets a certain degree of compression and the shift process is in stage 2 or stage 3 .

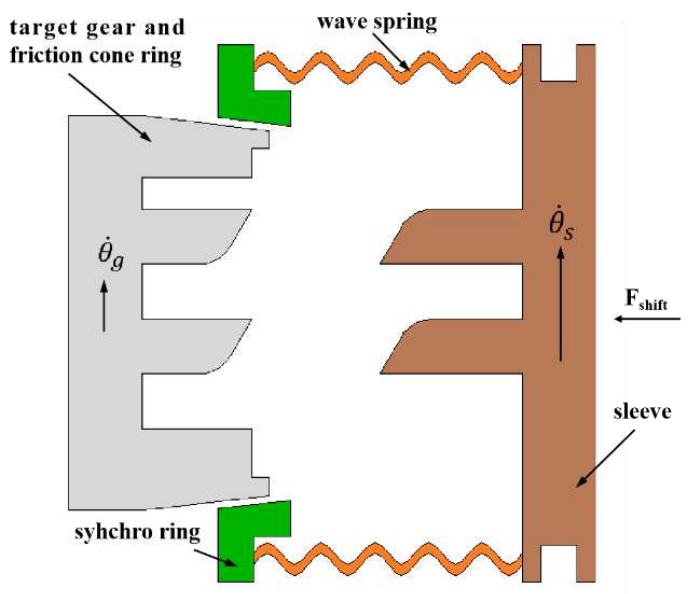

Fig.4. Schematic diagram of the structure of the sleeve in the middle position of the synchronizer

$$
\begin{gathered}
I_{1} \ddot{\theta}_{1}=T_{1}-c_{1} \dot{\theta}_{1}-c_{2}\left(\dot{\theta}_{1}-\dot{\theta}_{s}\right)-k_{2}\left(\theta_{1}-\theta_{s}\right) \\
\left(I_{s}+I_{r}\right) \ddot{\theta}_{s}=c_{2}\left(\dot{\theta}_{1}-\dot{\theta}_{s}\right)+k_{2}\left(\theta_{1}-\theta_{s}\right) \\
I_{g} \ddot{\theta}_{g}=-c_{3} \dot{\theta}_{g}-R_{g}\left[c_{m}\left(\dot{y}_{01}+\dot{y}_{g}-\dot{y}_{x}-\dot{e}_{y 1}\right)+\right. \\
\left.k_{m}\left(y_{01}+y_{g}-y_{x}-e_{y 1}\right)\right] \\
\left(I_{x}+I_{e}\right) \ddot{\theta}_{x}=-c_{4} \dot{\theta}_{x}+R_{x}\left[\begin{array}{c}
c_{m}\left(\dot{y}_{01}+\dot{y}_{g}-\dot{y}_{x}-\dot{e}_{y 1}\right) \\
+k_{m}\left(y_{01}+y_{g}-y_{x}-e_{y 1}\right)
\end{array}\right]- \\
R_{e}\left[\begin{array}{c}
c_{m}\left(\dot{y}_{02}+\dot{y}_{e}-\dot{y}_{f}-\dot{e}_{y 2}\right) \\
+k_{m}\left(y_{02}+y_{e}-y_{f}-e_{y 2}\right)
\end{array}\right] \\
I_{f} \ddot{\theta}_{f}=R_{f}\left[\begin{array}{c}
c_{m}\left(\dot{y}_{02}+\dot{y}_{e}-\dot{y}_{f}-\dot{e}_{y 2}\right) \\
+k_{m}\left(y_{02}+y_{e}-y_{f}-e_{y 2}\right)
\end{array}\right]-c_{0}\left(\dot{\theta}_{f}-\dot{\theta}_{v}\right)- \\
k_{0}\left(\theta_{f}-\theta_{v}\right) \\
I_{v} \ddot{\theta}_{v}=c_{0}\left(\dot{\theta}_{f}-\dot{\theta}_{v}\right)+k_{0}\left(\theta_{f}-\theta_{v}\right)-T_{v}
\end{gathered}
$$

Where $I_{s}$ is the moment of inertia of the hub and the sleeve. $I_{r}$ is the moment of inertia of the synchro ring. $\theta_{i}$ is the rotation angle of the corresponding component, $\dot{\theta}_{i}$ is the angular velocity of the corresponding component, $\ddot{\theta}_{i}$ is the angular acceleration of the corresponding component, where $i=\mathrm{s}, \mathrm{g}, \mathrm{x}, \mathrm{f}, \mathrm{v}, 1 . R_{j}$ is the basic radius of the corresponding gear, $y_{j}$ are the translational displacement of the corresponding gear. $\dot{y}_{j}$ are the translational velocity of the corresponding gear, where $j=\mathrm{g}, \mathrm{x}, \mathrm{e}, \mathrm{f} . y_{01}$ and $y_{02}$ are the relative displacement of meshing gear. $\dot{y}_{01}$ and $\dot{y}_{02}$ are the relative velocity of meshing gear. $e_{y 1}, e_{y 2}$ are the static transmission error of the gear pair. $\dot{\mathrm{e}}_{y l}, \dot{\mathrm{e}}_{y 2}$ are the static transmission velocity error of the gear pair. $F_{\text {shift }}$ is the force of shifting.

Stage 2: Synchro ring and friction cone ring contact for synchronization.

The state of PCFRS at this stage is shown in Fig. 5. The sleeve stops moving in the axial direction, and the spring 
force generated by the spring compression makes the friction cone surface of the synchro ring and the friction cone ring keep in touch. The friction surface will be produced friction torque, because there is a speed difference between the synchro ring and the friction cone ring. The synchro ring is constrained by the hub, so that the rotational speed of the PCFRS and the input shaft gradually become consistent. At this stage, the shift motor stops driving, the shift actuator adopts the form of a worm gear, and the stop of the sleeve is realized by the self-locking characteristic of the worm gear. When the speed difference is reached the allowable teeth-entering range by the friction torque, this stage ends and the shifting process will proceed to stage 3 .
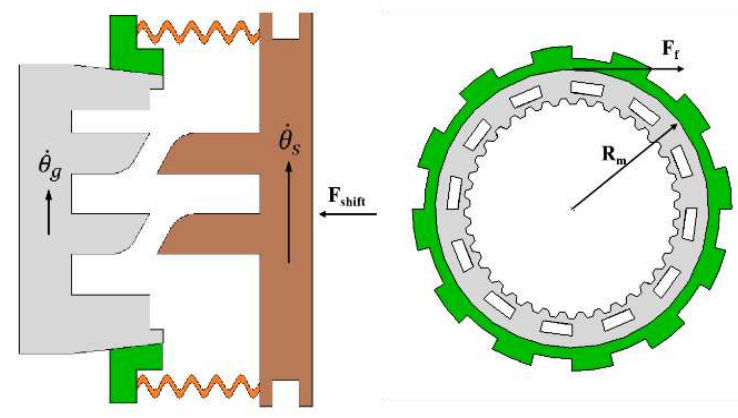

Fig.5. Schematic diagram of the synchronization process of the synchro ring and the friction cone ring contacting

$$
\begin{gathered}
I_{1} \ddot{\theta}_{1}=T_{1}-c_{1} \dot{\theta}_{1}-c_{2}\left(\dot{\theta}_{1}-\dot{\theta}_{s}\right)-k_{2}\left(\theta_{1}-\theta_{s}\right) \\
\left(I_{s}+I_{r}\right) \ddot{\theta}_{s}=c_{2}\left(\dot{\theta}_{1}-\dot{\theta}_{s}\right)+k_{2}\left(\theta_{1}-\theta_{s}\right)-F_{f} R_{m} \\
I_{g} \ddot{\theta}_{g}=F_{f} R_{m}-c_{3} \dot{\theta}_{g}-R_{g}\left[c_{m}\left(\dot{y}_{01}+\dot{y}_{g}-\dot{y}_{x}-\dot{e}_{y 1}\right)+\right. \\
\left.k_{m}\left(y_{01}+y_{g}-y_{x}-e_{y 1}\right)\right] \\
\left(I_{x}+I_{e}\right) \ddot{\theta}_{x}=-c_{4} \dot{\theta}_{x}+R_{x}\left[\begin{array}{c}
c_{m}\left(\dot{y}_{01}+\dot{y}_{g}-\dot{y}_{x}-\dot{e}_{y 1}\right) \\
+k_{m}\left(y_{01}+y_{g}-y_{x}-e_{y 1}\right)
\end{array}\right] \\
-R_{e}\left[\begin{array}{c}
c_{m}\left(\dot{y}_{02}+\dot{y}_{e}-\dot{y}_{f}-\dot{e}_{y 2}\right) \\
+k_{m}\left(y_{02}+y_{e}-y_{f}-e_{y 2}\right)
\end{array}\right] \\
I_{f} \ddot{\theta}_{f}=R_{f}\left[\begin{array}{c}
c_{m}\left(\dot{y}_{02}+\dot{y}_{e}-\dot{y}_{f}-\dot{e}_{y 2}\right) \\
+k_{m}\left(y_{02}+y_{e}-y_{f}-e_{y 2}\right)
\end{array}\right]-c_{0}\left(\dot{\theta}_{f}-\dot{\theta}_{v}\right)- \\
k_{0}\left(\theta_{f}-\theta_{v}\right) \\
I_{v} \ddot{\theta}_{v}=c_{0}\left(\dot{\theta}_{f}-\dot{\theta}_{v}\right)+k_{0}\left(\theta_{f}-\theta_{v}\right)-T_{v}
\end{gathered}
$$

Where $F_{f}$ is the friction force between the synchronizer ring and the friction cone ring. $R_{m}$ is the friction equivalent radius.

Stage 3: Before the speed difference is eliminated, the sleeve enters the teeth and the friction cone ring teeth contact.

The state of PCFRS at this stage is shown in Fig. 6. After the synchronization stage is completed, the sleeve continues to be moved. According to the shift characteristics of the PCFRS, the sleeve can move again without completely eliminating the speed difference. When the speed difference reaches the allowable range, the sleeve moves to contact the internal teeth of the friction cone ring to turn the teeth, and the contact between the engagement sleeve and the friction cone ring generates surface contact force. At this time, there is a friction torque between the friction cone ring and the synchro ring, and there is also a turn-teeth torque between the friction cone ring and the sleeve. Both friction torque and turn-teeth torque promote the elimination of the speed difference. Under the action of the helical teeth, the sleeve moves to the internal teeth hole of the friction cone ring. At the end of this phase, the shift process proceeds to stage 4 or stage 5 .

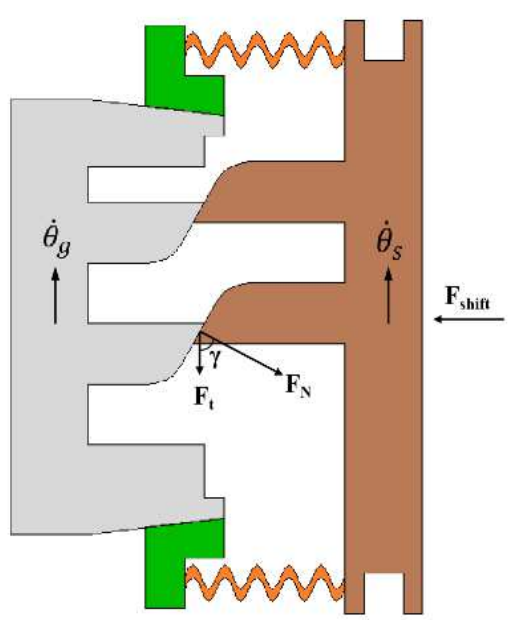

Fig.6. Schematic diagram of the structure of the sleeve and the friction cone ring contacting

$$
\begin{gathered}
I_{1} \ddot{\theta}_{1}=T_{1}-c_{1} \dot{\theta}_{1}-c_{2}\left(\dot{\theta}_{1}-\dot{\theta}_{s}\right)-k_{2}\left(\theta_{1}-\theta_{s}\right) \\
\left(I_{s}+I_{r}\right) \ddot{\theta}_{s}=c_{2}\left(\dot{\theta}_{1}-\dot{\theta}_{s}\right)+k_{2}\left(\theta_{1}-\theta_{s}\right)-F_{f} R_{m}-F_{t} R_{t} \\
I_{g} \ddot{\theta}_{g}=F_{t} R_{t}+F_{f} R_{m}-c_{3} \dot{\theta}_{g}-R_{g}\left[c _ { m } \left(\dot{y}_{01}+\dot{y}_{g}-\dot{y}_{x}-\right.\right. \\
\left.\left.\dot{e}_{y 1}\right)+k_{m}\left(y_{01}+y_{g}-y_{x}-e_{y 1}\right)\right] \\
\left(I_{x}+I_{e}\right) \ddot{\theta}_{x}=-c_{4} \dot{\theta}_{x}+R_{x}\left[\begin{array}{c}
c_{m}\left(\dot{y}_{01}+\dot{y}_{g}-\dot{y}_{x}-\dot{e}_{y 1}\right) \\
+k_{m}\left(y_{01}+y_{g}-y_{x}-e_{y 1}\right)
\end{array}\right]- \\
R_{e}\left[\begin{array}{c}
c_{m}\left(\dot{y}_{02}+\dot{y}_{e}-\dot{y}_{f}-\dot{e}_{y 2}\right) \\
\left.+k_{m}\left(y_{02}+y_{e}-y_{f}-e_{y 2}\right)\right]
\end{array}\right. \\
I_{f} \ddot{\theta}_{f}=R_{f}\left[\begin{array}{c}
c_{m}\left(\dot{y}_{02}+\dot{y}_{e}-\dot{y}_{f}-\dot{e}_{y 2}\right) \\
+k_{m}\left(y_{02}+y_{e}-y_{f}-e_{y 2}\right)
\end{array}\right]-c_{0}\left(\dot{\theta}_{f}-\dot{\theta}_{v}\right)- \\
k_{0}\left(\theta_{f}-\theta_{v}\right) \\
I_{v} \ddot{\theta}_{v}=c_{0}\left(\dot{\theta}_{f}-\dot{\theta}_{v}\right)+k_{0}\left(\theta_{f}-\theta_{v}\right)-T_{v}
\end{gathered}
$$

Where $F_{t}$ is the tangential component of the turn-teeth force. $F_{N}$ is the positive pressure on the bevel. $R_{t}$ is the turn-teeth radius. $\gamma$ is the teeth surface angle.

Stage 4: After the speed is eliminated, the sleeve teeth moves in contact with the friction cone ring teeth.

The state of PCFRS at this stage is shown in Fig. 7. This situation exists when the speed difference between the sleeve and the friction cone ring is eliminated, but the sleeve has not been fully meshed. Therefore, stage 4 is 
required. The sleeve continues to turn the friction cone ring teeth and advance the teeth. The gear shifting method at this stage is that the sleeve teeth are slowly advanced so that the teeth surface is in continuous contact, so that no excessive great speed difference is generated, and finally a successful meshing without a speed difference is achieved.

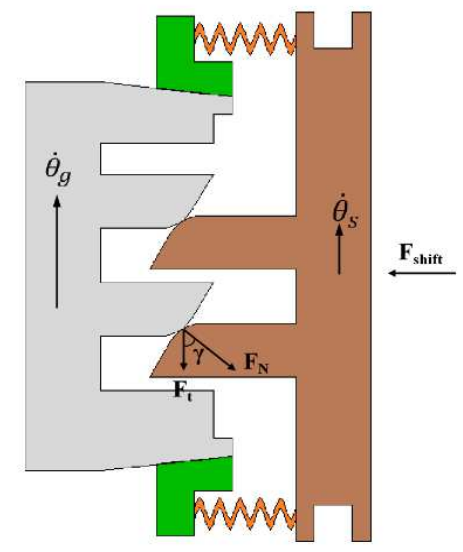

Fig.7. Schematic diagram of the turning teeth after the sleeve teeth and the friction cone ring teeth contacting

$$
\begin{gathered}
I_{1} \ddot{\theta}_{1}=T_{1}-c_{1} \dot{\theta}_{1}-c_{2}\left(\dot{\theta}_{1}-\dot{\theta}_{s}\right)-k_{2}\left(\theta_{1}-\theta_{s}\right) \\
\left(I_{s}+I_{r}\right) \ddot{\theta}_{s}=c_{2}\left(\dot{\theta}_{1}-\dot{\theta}_{s}\right)+k_{2}\left(\theta_{1}-\theta_{s}\right)+F_{f} R_{m}-F_{t} R_{t} \\
I_{g} \ddot{\theta}_{g}=F_{t} R_{t}-F_{f} R_{m}-c_{3} \dot{\theta}_{g}-R_{g}\left[c _ { m } \left(\dot{y}_{01}+\dot{y}_{g}-\dot{y}_{x}-\right.\right. \\
\left.\left.\dot{e}_{y 1}\right)+k_{m}\left(y_{01}+y_{g}-y_{x}-e_{y 1}\right)\right] \\
\left(I_{x}+I_{e}\right) \ddot{\theta}_{x}=-c_{4} \dot{\theta}_{x}+R_{x}\left[\begin{array}{c}
c_{m}\left(\dot{y}_{01}+\dot{y}_{g}-\dot{y}_{x}-\dot{e}_{y 1}\right) \\
+k_{m}\left(y_{01}+y_{g}-y_{x}-e_{y 1}\right)
\end{array}\right]- \\
R_{e}\left[\begin{array}{c}
c_{m}\left(\dot{y}_{02}+\dot{y}_{e}-\dot{y}_{f}-\dot{e}_{y 2}\right) \\
+k_{m}\left(y_{02}+y_{e}-y_{f}-e_{y 2}\right)
\end{array}\right] \\
I_{f} \ddot{\theta}_{f}=R_{f}\left[\begin{array}{c}
c_{m}\left(\dot{y}_{02}+\dot{y}_{e}-\dot{y}_{f}-\dot{e}_{y 2}\right) \\
+k_{m}\left(y_{02}+y_{e}-y_{f}-e_{y 2}\right)
\end{array}\right]-c_{0}\left(\dot{\theta}_{f}-\dot{\theta}_{v}\right)- \\
k_{0}\left(\theta_{f}-\theta_{v}\right) \\
I_{v} \ddot{\theta}_{v}=c_{0}\left(\dot{\theta}_{f}-\dot{\theta}_{v}\right)+k_{0}\left(\theta_{f}-\theta_{v}\right)-T_{v}
\end{gathered}
$$

Stage 5: After the speed is eliminated, the contact between the sleeve and the teeth surface of the friction cone produces a speed difference. Use the generated speed difference to complete the teeth advancement.

The state of PCFRS at this stage is shown in Fig. 8. It exists in the situation of stage 4 , but is not the slow gear advancement. The teeth advancement according to the teeth advancement state of stage 3, resulting in the speed difference, and then the slow teeth advancement is used. The difference in speed reduces the relative angle between the sleeve and the teeth hole of the friction cone ring to realize shifting. At this stage, there may be impact during meshing, but the difference in speed is small, and the impact produced is within an acceptable range. In addition, the speed difference can also be eliminated when the teeth is advanced to realize the speed difference-free shift.

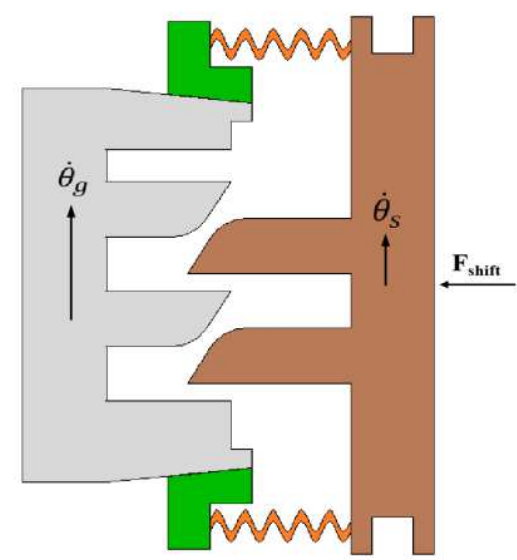

Fig.8. Schematic diagram of the structure of using the speed difference generated by the turn-teeth to complete the shifting

$$
\begin{gathered}
I_{1} \ddot{\theta}_{1}=T_{1}-c_{1} \dot{\theta}_{1}-c_{2}\left(\dot{\theta}_{1}-\dot{\theta}_{s}\right)-k_{2}\left(\theta_{1}-\theta_{s}\right) \\
\left(I_{s}+I_{r}\right) \ddot{\theta}_{s}=c_{2}\left(\dot{\theta}_{1}-\dot{\theta}_{s}\right)+k_{2}\left(\theta_{1}-\theta_{s}\right)+F_{f} R_{m} \\
I_{g} \ddot{\theta}_{g}=-F_{f} R_{m}-c_{3} \dot{\theta}_{g}-R_{g}\left[c_{m}\left(\dot{y}_{01}+\dot{y}_{g}-\dot{y}_{x}-\dot{e}_{y 1}\right)+\right. \\
\left.k_{m}\left(y_{01}+y_{g}-y_{x}-e_{y 1}\right)\right] \\
\left(I_{x}+I_{e}\right) \ddot{\theta}_{x}=-c_{4} \dot{\theta}_{x}+R_{x}\left[\begin{array}{c}
c_{m}\left(\dot{y}_{01}+\dot{y}_{g}-\dot{y}_{x}-\dot{e}_{y 1}\right) \\
+k_{m}\left(y_{01}+y_{g}-y_{x}-e_{y 1}\right)
\end{array}\right]- \\
R_{e}\left[\begin{array}{c}
c_{m}\left(\dot{y}_{02}+\dot{y}_{e}-\dot{y}_{f}-\dot{e}_{y 2}\right) \\
+k_{m}\left(y_{02}+y_{e}-y_{f}-e_{y 2}\right)
\end{array}\right] \\
I_{f} \ddot{\theta}_{f}=R_{f}\left[\begin{array}{c}
c_{m}\left(\dot{y}_{02}+\dot{y}_{e}-\dot{y}_{f}-\dot{e}_{y 2}\right) \\
+k_{m}\left(y_{02}+y_{e}-y_{f}-e_{y 2}\right)
\end{array}\right]-c_{0}\left(\dot{\theta}_{f}-\dot{\theta}_{v}\right)- \\
k_{0}\left(\theta_{f}-\theta_{v}\right) \\
I_{v} \ddot{\theta}_{v}=c_{0}\left(\dot{\theta}_{f}-\dot{\theta}_{v}\right)+k_{0}\left(\theta_{f}-\theta_{v}\right)-T_{v}
\end{gathered}
$$

The state of PCFRS is random, and it is divided into the above five representative stages. In addition, the PCFRS engages the sleeve and the friction cone during the shifting process. The relative angle of the friction cone ring teeth hole is also random, and there may be a small or zero relative angle to the friction cone ring teeth hole during the teeth engagement process, so that the shift is completed directly after the gear is dialed or the gear is directly engaged.

\section{Simulation analysis of the synchronization effect}

In order to simplify the simulation process, the target gear replaces the gear meshing system in the transmission as the output shaft, converts the wheel inertia to the target gear, and sets the specified initial speed and moment of inertia for the target gear, the input shaft and the synchronizer. Combining the structural characteristics of the PCFRS, firstly, the PID position controller is used to move the sleeve to the specified position, and after synchronization is completed, another PID speed controller is used to control the shift motor to realize shifting. 


\subsection{Simulation results analysis of the shifting process}

The shifting process of PCFRS is shown in Fig. 9, which shows the relationship between the rotational speed of the sleeve and the target gear at all stages of the shifting. Because the moment of inertia of the input shaft is smaller than the output shaft during the shifting, it can be seen from the figures that the speed of the target gear is difficult to change, so the speed of the input shaft changes quickly during the shift to promote the shift. Therefore, when analyzing the simulation results, the rotation speed of the input shaft and the displacement of the sleeve on the input shaft is mainly analyzed.

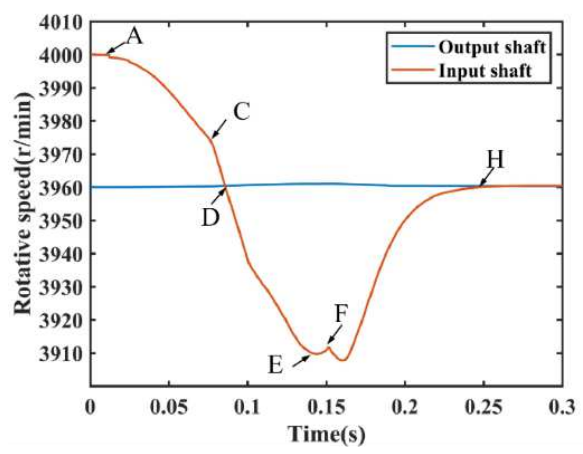

(a) Speed curves of the input shaft and the output shaft

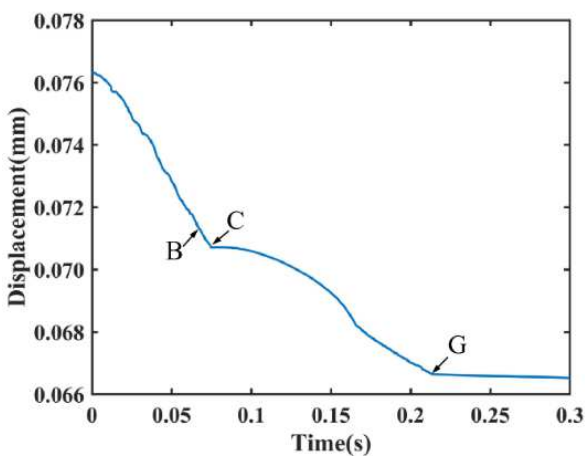

(b) Displacement curve of the sleeve

Fig.9. Transmission movement state during the shift

Combining the rotational speed curves of the input shaft and the displacement curve of the sleeve, point $\mathrm{A}$ in the figure is that the gap between the components of the shift process is eliminated and the idle stroke of the sleeve moving in the direction of the target gear is completed. As the movement of the sleeve enters the synchronization phase, point $\mathrm{B}$ indicates that the sleeve to reach the set position, and it is determined that the speed difference has reached the range of sleeve advancement, and then the sleeve advancement continues. Point $\mathrm{C}$ indicates that the sleeve is in contact with the friction cone ring teeth while moving. Then it enters the turn-teeth phase and the teeth surface contact in the gear shifting stage produces a force that is opposite to the speed directions of the sleeve and the friction cone ring to accelerate the synchronization process. The synchronization is completed at point $\mathrm{D}$, and then the sleeve is moved at a certain speed. At point E, the sleeve teeth are separated from the friction cone ring teeth, and the friction torque continues to be synchronized while the engagement sleeve is moving. At point $F$, the sleeve teeth contacts the friction cone ring gear again, and after the contact continues to turn teeth, which produces a speed difference and the teeth separate. The sleeve teeth still advances at a certain speed, and the friction torque quickly eliminates the speed difference. At point G, the advancement of the sleeve is completed, and there is still a small speed difference at this time. Due to the small gap between the sleeve teeth and the teeth groove of the friction cone ring, synchronization is still performed after the advancement of sleeve is completed. At point $\mathrm{H}$, the speed difference is completely eliminated, achieving no speed difference shifting.

\subsection{Shift quality analysis}

This section will conduct simulation analysis based on the established dynamics models. The PCFRS studied in this paper is always in a compressed state during the shifting process, and the spring compression generates pressure, resulting in friction torque that directly affects the sliding work during the shifting process and even affects the working conditions of the synchronizer. Therefore, this section will verify whether the sliding work is reasonable and whether it affects the work. In addition, to study the shift performance of the PCFRS, the factors that should also be considered in the shift process are shift impact and shift time.

Based on the above considerations, the shift impact, sliding work, and shift time of the PCFRS will be analyzed in detail to verify the rationality of the mechanism.

\subsubsection{Shift impact analysis}

The PCFRS mentioned in this article can realize zero-speed difference shift during shifting, so as to realize the shift effect without impact. However, if the speed difference is made within a reasonable range, the shift time will be shorter. This section will discuss the relationship between the impact caused by the different speed difference between the sleeve and the target gear. Analyze the impact degree generated by shifting with different speed differences.

Impact is an important indicator for evaluating shift quality. The formula is expressed as follows.

$$
j=\frac{d^{2} v}{3.6 d t^{2}}=\frac{d a}{d t}=\frac{d \alpha}{d t} R_{w}=\frac{d T_{w}}{I_{v} d t} R_{w}
$$

where $v$ is the longitudinal speed of vehicle. $a$ represents the longitudinal velocity of the vehicle. $\alpha$ is the wheel angular acceleration. $T_{w}$ is the wheel driving torque. $I_{v}$ is the moment of inertia of the wheel. $R_{w}$ is the wheel radius. 
In real situations, shift will produce impact, and flexible components such as tires will cushion the impact. In the simulation process, the flexible components are not taken into consideration, so the impact value obtained by simulation will deviate greatly from the true value. In addition, the impact is expressed by the second derivative of the longitudinal speed of the vehicle [31]. The software realizes the shift simulation with a certain simulation step length, so it is not exactly the same as the actual situation, and the simulation step length will affect the rate of change of the real curve. The second derivation of the curve will make the deviation larger, and even the deviation will cause the simulation result to be inconsistent with the actual situation. The degree of impact can also be calculated by derivation of the wheel torque. The wheel torque is the torque transmitted to the wheel by the input shaft torque after the engagement sleeve teeth contact the internal teeth of the friction cone ring. At the moment when the sleeve teeth contacts the internal teeth of the friction cone ring, the impact torque is also generated by the teeth surface collision and the generation time is very short, which can reflect the impact characteristics, so it is also meaningful to measure the shift impact. In addition, the impact torque only needs to derive the longitudinal velocity once, and the resulting error will be smaller. This paper will analyze the influence of simulation step length on wheel torque and impact, and verify the influence of simulation step length and non-flexible parts on the results. Choose the ILRS simulation model that is easier to observe for verification. Fig. 10 shows the values of wheel torque and impact.

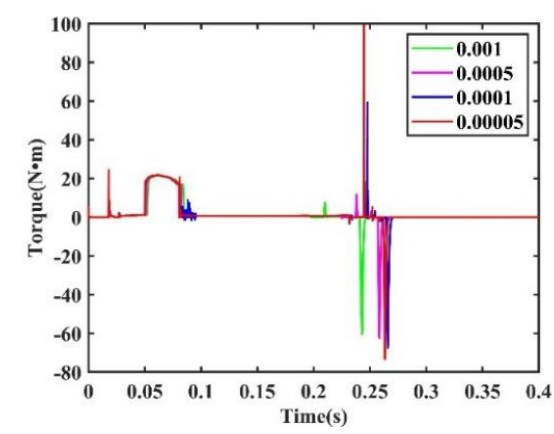

(a) Drive torque curves

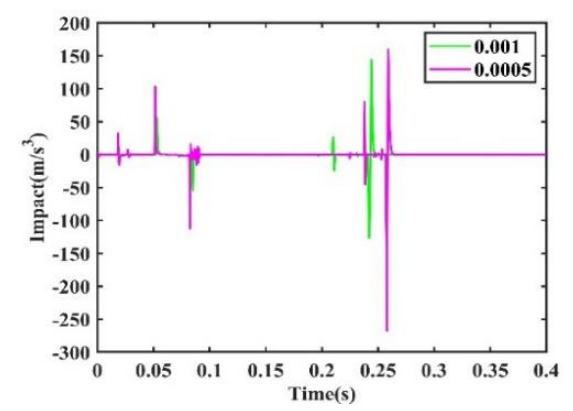

(b) Impact curves

Fig.10. Curves of the drive torque and the impact obtained by simulation of different simulation steps
For the convenience of observation, only two sets of simulations with similar wheel torques and similar simulation steps are selected for comparison in the impact curve in the figure. It can be seen from the figure that the simulation step length not only affects the size of the simulation value, but also causes a deviation in the time for the final completion of the shift. The simulation step size has little effect on the wheel torque value when the final engagement sleeve teeth are in contact with the internal teeth of the friction cone ring, but the impact curve varies greatly with the simulation step size. It can be seen that the value of the impact obtained by simulation is not stable. If the impact of the PCFRS is compared with the simulation result of the ILRS, the result is difficult to guarantee the truth, so this paper will take two measures. The impact torque produced by this synchronizer is compared to highlight the characteristics of the mechanism. Therefore, this paper will compare the impact torque produced by the two synchronizers to highlight the characteristics of the mechanism.

The output torque that only needs to be derived from the longitudinal speed of the vehicle once to replace the impact, which can reflect the gear shift impact and reduce the simulation deviation as shown at below.

$$
T_{w}=\frac{I_{v} d v}{R_{w} d t}
$$

Using the impact torque instead of the impact evaluates shift quality, the torque is expressed as follows:

$$
T_{w}=I_{v} \cdot \alpha=I_{v} \cdot \frac{\alpha_{2}}{i_{0}}
$$

where $\alpha_{2}$ is the angular acceleration of input shaft. $i_{0}$ is the gear ratio of the transmission.

As shown in Fig. 11, different shifting effects with different moving speeds of the sleeve are adopted, and it takes a long time to achieve a shift without a speed difference of $0.2406 \mathrm{~s}$, but the impact torque of teeth collision is not generated; When the shift produces the impact torque of $66.5673 \mathrm{~N} \cdot \mathrm{m}$ in the shift mode, the shift time is only $0.2031 \mathrm{~s}$. This result will be compared with the shift simulation results of the ILRS in section 5.

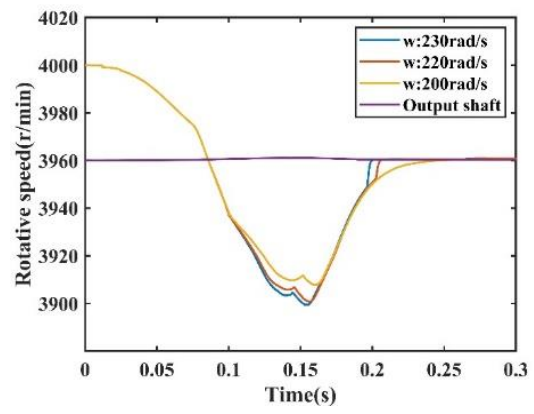

(a) The speed curves of the input shaft and the output shaft with different moving speeds of the sleeve 


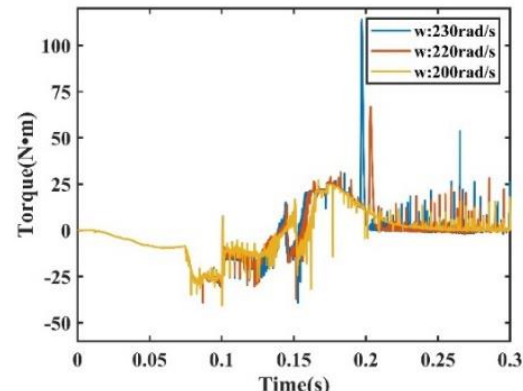

(b) Impact torques of different shift effects and target gear output speed

Fig.11. Shift effect of PCFRS at different sleeve moving speeds

\subsubsection{Sliding power analysis}

The PCFRS shifts through the friction torque generated by the contact between the synchro ring and the friction cone ring to eliminate the speed difference and realizes the shift. Moreover, the force generated by the compression of the wave spring is applied to the synchro ring, so the sliding friction work during the shifting process cannot be ignored. The sliding friction work is mainly expressed in the form of heat, which directly affects the reliability of the synchronizer. It may even cause irreversible damage to the synchronizer and affect its lifespan. This section will verify whether the shift mode meets the requirements. According to the definition of sliding work, sliding work $L_{f}$ can be expressed as follows:

$$
L_{f}=\int_{0}^{\theta} T_{f} d \theta
$$

From formula(35), $T_{f}$ is:

$$
T_{f}=\frac{F_{a} f R_{m}}{\sin \beta}
$$

where $F_{a}$ is the axial contact force of the friction cone ring and the synchronizer ring. $f$ is the friction coefficient of friction cone and synchro ring friction pair. $\theta$ is the relative rotation angle of the friction cone ring relative to the friction cone surface of the target gear during the synchronous friction process, $d \theta=\left|\omega_{g}-\omega_{r}\right| d t . \beta$ is the angle of friction cone. The analysis of the sliding work formula is[32]:

$$
L_{f}=\int_{0}^{t_{T}} \frac{\pi F_{a} f R_{m}}{30 \sin \beta}\left|\omega_{g}-\omega_{r}\right| d t
$$

where $t_{T}$ is the relative sliding time of friction cone ring and synchro ring. $\omega_{g}, \omega_{r}$ are the rotation speed of the target gear and the synchro ring.

Due to the difference of the synchronizers, the shift processes and the synchronizer friction cone rings are also different. In order to make the evaluation more objective, the specific sliding work and specific sliding power are introduced to evaluate the performance.

The specific sliding work is expressed as follows:

$$
q=\frac{L_{f}}{A_{f}}
$$

where $A_{f}$ is the contact area of the friction cone and the synchro ring.
In the synchronization stage, the instantaneous specific sliding power is used. The calculation can be calculated by dividing the contact area by the product of the friction torque and the relative angular velocity difference, which is expressed as follows:

$$
q_{p}=\frac{\pi T_{f} \cdot\left|\omega_{g}-\omega_{r}\right|}{30 A_{f}}
$$

Fig. 12 shows the specific sliding work and specific sliding power between the friction elements during the shifting process. The allowable values of specific sliding work and specific sliding power are $0.09 \mathrm{~J} / \mathrm{mm}^{2}$ and 0.45 $\mathrm{W} / \mathrm{mm}^{2}[33]$.

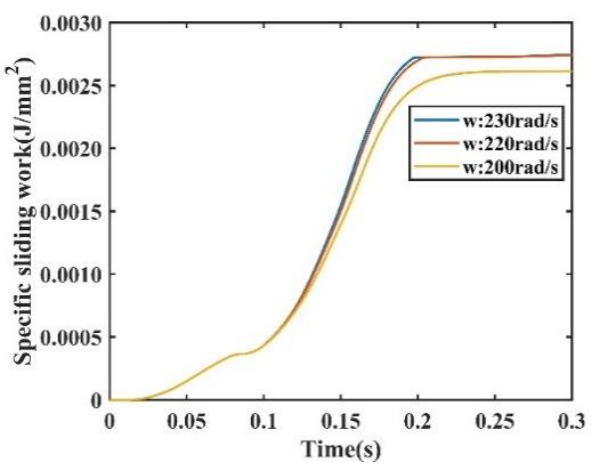

(a) Specific sliding work curves

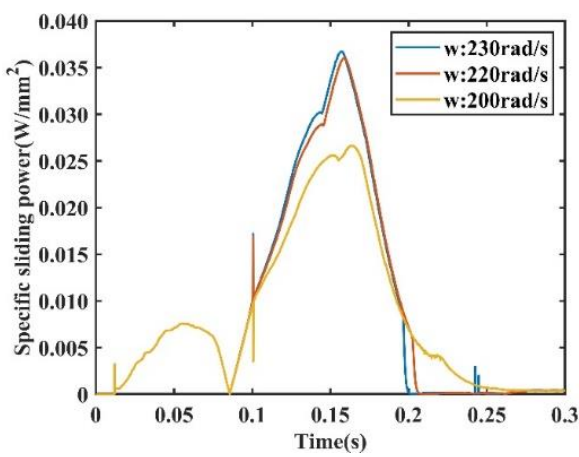

(b) Specific sliding power curves

Fig.12. The specific sliding work and specific sliding power of the PCFRS under different shift speeds

It can be seen from the figure that the specific sliding work and specific sliding power of the PCFRS in different shift modes meet the allowable value.

\subsubsection{PV value analysis}

Regarding the working conditions of the synchronizer, the PV value is often used to check[34], that is, the product value of the surface pressure of the friction cone and the relative sliding speed. The PV value can intuitively reflect whether the shifting force and speed difference set during the shifting process are reasonable.

The surface pressure $\mathrm{P}$ of the PV value is expressed as:

$$
P=\frac{F_{a}}{\sin \beta \cdot A_{f}}
$$

The relative sliding speed $\mathrm{V}$ of the PV value is 


$$
V=\frac{100 \pi \cdot \mathrm{R}_{m} \cdot\left|\omega_{g}-\omega_{r}\right|}{3}
$$

Organizing the formulas (40) and (41) get the following formula:

$$
P V=\frac{100 F_{a} \cdot \pi \cdot R_{m} \cdot\left|\omega_{g}-\omega_{r}\right|}{3 \sin \alpha \cdot A_{f}}<[P][V]
$$

According to ZF Friedrichshafen AG's recommendation, the PV value should not be greater than $15000 \mathrm{~N} /(\mathrm{mm} \bullet \mathrm{s})$ [33]. The simulation curve is shown in Fig. 13.

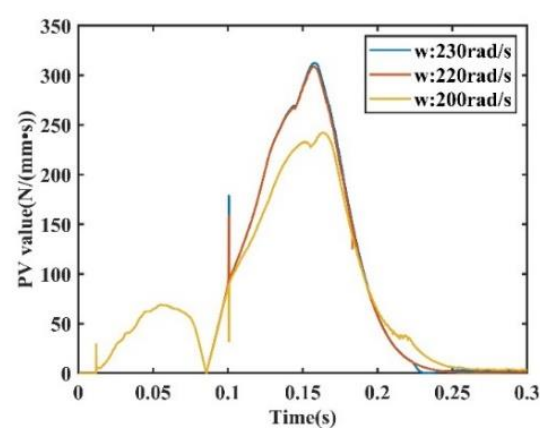

Fig.13. PV value curves of the PCFRS

It can be seen from the figure that when the sleeve moves at different speeds, the working conditions of the synchronizer all meet the allowable value and meet the requirements of use. The simulation of the above shifting modes is reasonable.

\section{Synchronization performance comparison verification}

In this chapter, the ILRS is modeled and simulated, and the same control method and parameters as the PCFRS are used for the simulation of shifting with the same speed difference. Table 1 shows the main parameters of the simulation of the PCFRS and the ILRS.

Table 1. Main parameters of the synchronizers

\begin{tabular}{ccc}
\hline Symbol & Unit & Value \\
\hline$I_{\mathrm{v}}$ & $\mathrm{kg} \cdot \mathrm{m}^{2}$ & 78 \\
$R_{\mathrm{w}}$ & $\mathrm{m}$ & 0.266 \\
$R_{\mathrm{m}}$ & $\mathrm{m}$ & 0.025 \\
$i_{0}$ & 1 & 5.2542 \\
$f$ & 1 & 0.11 \\
$\beta$ & $\circ$ & 6.5 \\
$A_{\mathrm{f}}$ & $\mathrm{mm}^{2}$ & 628.319 \\
\hline
\end{tabular}

\subsection{Modelling and simulation analysis of ILRS}

The ILRS is different from the PCFRS. The ILRS is unable to eliminate the speed difference caused by the engagement of the shift. The PCFRS can eliminate the speed difference caused by the turn teeth, so PID speed control is used in combination with its characteristics; if the ILRS also uses PID speed control, in order to achieve the specified moving speed, the shifting force will produce a large speed difference and cause the large impact. Therefore, combined with the characteristics of the ILRS, the position control of the PID is used to realize the shifting force control with different $\mathrm{P}$ values, and different modes are used for multiple sets of simulations, which are designed to simply and intuitively reflect the mechanical characteristics of the ILRS. In addition, the shift effect that the ILRS can achieve makes the ILRS have compatibility with the corresponding mechanical characteristics of the PCFRS.

The shifting process of the ILRS is shown in Fig. 14, which shows the relationship between the input shaft speed and the output shaft speed at all stages when the ILRS shifts, and the displacement of the sleeve. Combined with the simulated speed curve and displacement curve, point A indicates that the ILRS completes the pre-synchronization phase and then enter the synchronization phase AB. The speed difference is eliminated at point B to complete synchronization. After the synchronization is completed, the synchro ring is turned in the $\mathrm{BC}$ section. At this time, the synchro ring and the target gear rotate at the same speed, and the turn-ring phase is completed at point $\mathrm{C}$. The sleeve continues to move to contact the target gear at point $\mathrm{D}$ and turns the target gear teeth. Point E is the collision between the teeth of sleeve and the teeth of the target gear. F point completes the movement of the sleeve to realize gear shift. It can be seen from the BEF stage that the speed difference produced by the ILRS during the turn-teeth phase will not disappear, and can only disappear through the collision between the teeth.

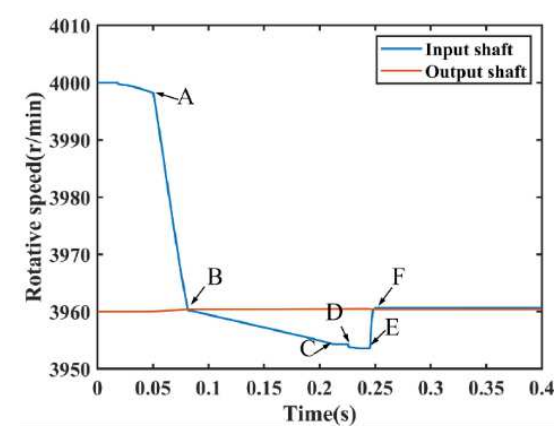

(a) Speed curves of the input shaft and the output shaft

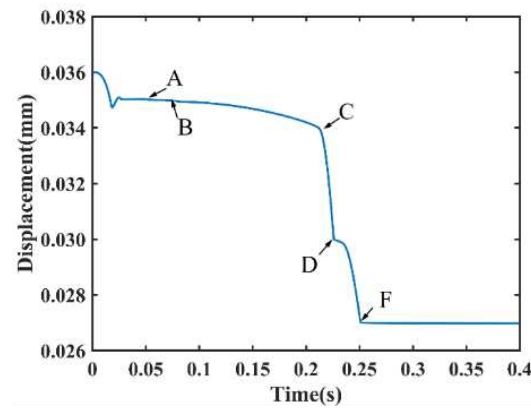

(b) Displacement curves of the sleeve

Fig.14. Transmission movement state during shift 


\subsection{Shifting impact of ILRS}

Since there is no relevant measurement standard for the value of the shift impact, this paper proposes a method to reflect the shift impact by comparing the impact torque. The shift simulation of the ILRS with different shifting forces and different shifting modes is carried out to show the rationality of the shift simulation of the ILRS in this paper and clearly reflects the mechanical characteristics of the ILRS. The simulation data of the ILRS is compared with the simulation data of the PCFRS, so as to highlight the advantages of the PCFRS in terms of impact. As shown in the figure, the PID position control is used to directly realize the shifting, and different $P$ values are used to realize different shifting forces. The smaller the $\mathrm{P}$ value, the smaller the shifting force produced.

It can be seen from Fig. 15 that as the shifting force decreases, the shifting time is longer, and the shifting impact torque also decreases.

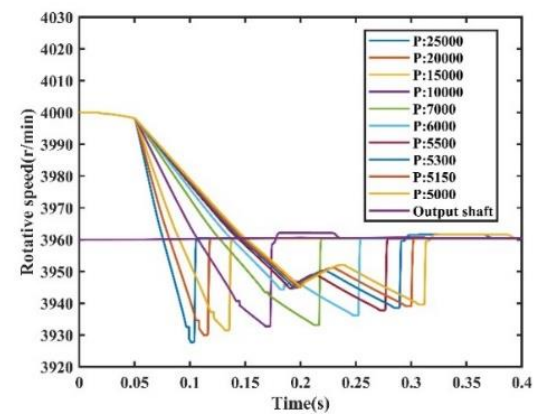

(a) The speeds relationship between the input shaft and the output shaft

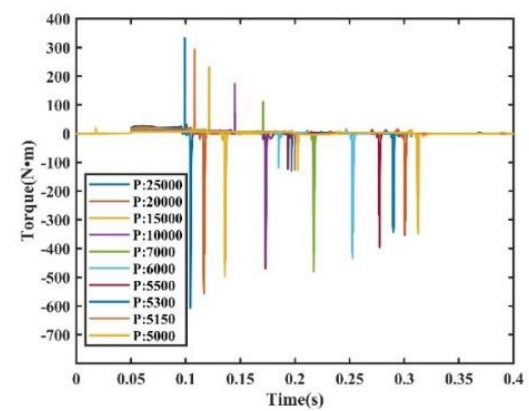

(b) Impact torque curves of different shift modes

Fig.15. Shift effect achieved by ILRS with single position control

It can be seen from the figure that in several sets of $\mathrm{P}$ value simulations, when the $P$ value is 20000 , the synchronization time is $0.0813 \mathrm{~s}$, which is close to the time of the PCFRS $0.0856 \mathrm{~s}$, so the $\mathrm{P}$ value for the second shift mode is 20000 . The second shift strategy is that when the speed difference between the sleeve and the target gear is eliminated, the shift force value achieved by the PID position controller is directly divided by different multiples to achieve a smaller gear shift force. The simulation results are shown in Fig. 16.

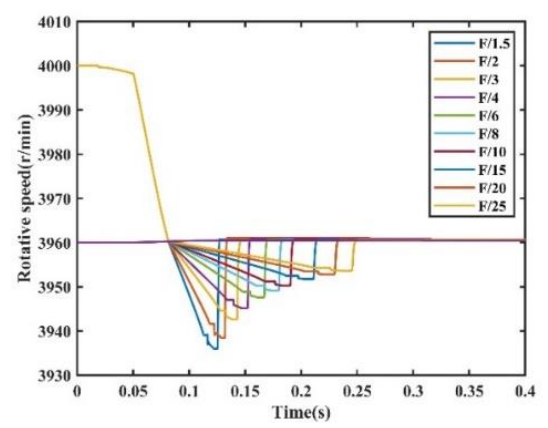

(a) The speeds relationship between the input shaft and the output shaft

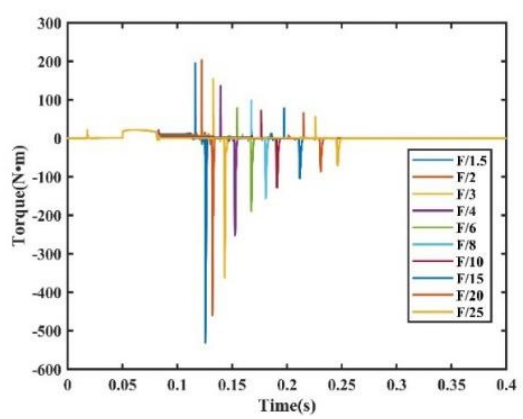

(b) Impact torque curves of different shift modes

Fig. 16. Shifting effect of ILRS adopts different shifting force after synchronization

The smaller the gear shifting force, the smaller the speed difference is produced by the shift, the longer the shift time will be. This shift strategy will produce a smaller impact torque compared to the pure position control. In the two shifting strategies, a group that produces an impact torque similar to that of the PCFRS is found, and the time required to shift is $0.2486 \mathrm{~s}$ when the impulsive torque is $69.8256 \mathrm{~N} \cdot \mathrm{m}$.

\subsection{Sliding work and PV value with ILRS shift}

The simulation of different strategies for the ILRS fully demonstrates its mechanical characteristics. However, while reflecting its performance, it also needs to consider the quality of its working conditions. The gear shift strategy also needs to ensure that the synchronizer wear is within a reasonable range to ensure the reliability, quality and service lifespan of the shift. Therefore, this section will verify whether the shifting process meets the standard through the sliding work and PV value of the ILRS calculated by simulation, so as to further determine the rationality of the ILRS shift simulation, which makes the comparison between the pressure-controllable friction ring synchronizer and the ILRS more convincing.

As shown in Fig. 17 and Fig. 18, they are the specific 
sliding work and specific sliding power generated during the shift simulation process of the two shift strategies.

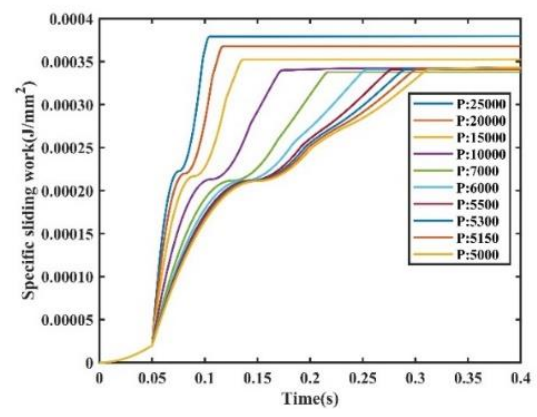

(a) Specific sliding work curves

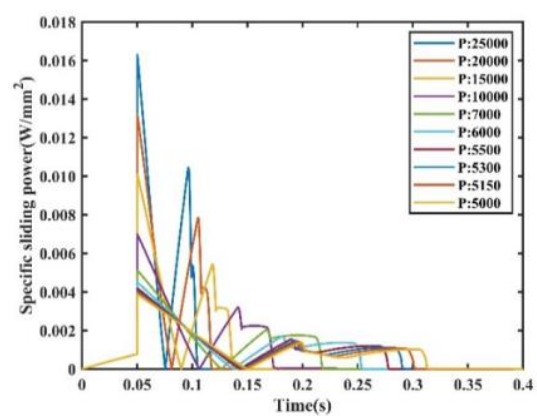

(b) Specific sliding power curves

Fig.17. Using different shifting forces to directly realize position-controlled shifting

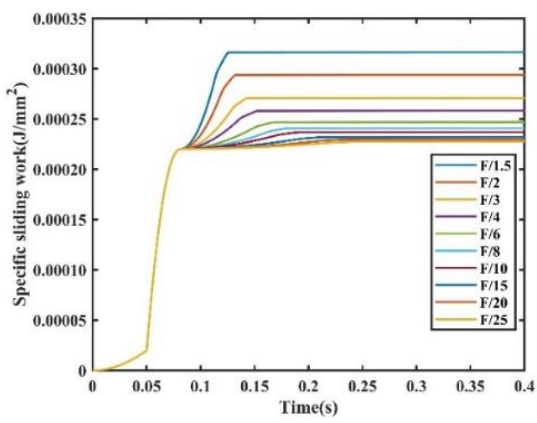

(a) Specific sliding work curves

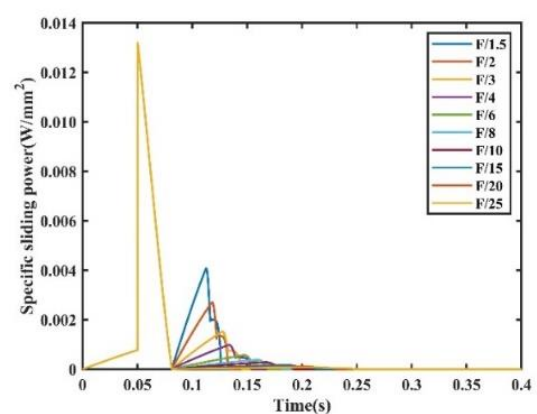

(b) Specific sliding power curves

Fig.18. Using different shifting forces after synchronization to achieve position-controlled shifting

It can be seen from the figures that the specific sliding work and specific sliding power are far less than the allowable value $0.09 \mathrm{~J} / \mathrm{mm}^{2}$ and $0.45 \mathrm{~W} / \mathrm{mm}^{2}$. This shows that the shift simulation of the ILRS in this paper meets the requirements of the mechanism, and the simulation data is reasonable.

As shown in Fig. 19, the PV values corresponding to the two shift strategies.

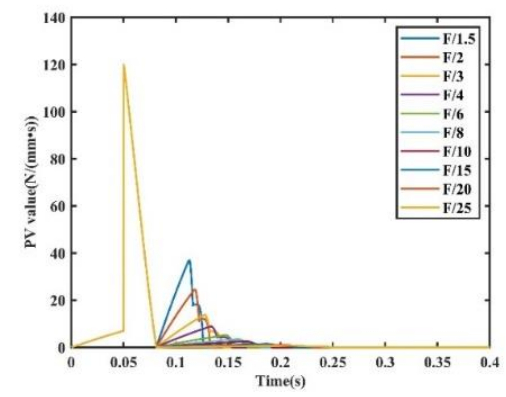

(a) PV value curves using direct position control

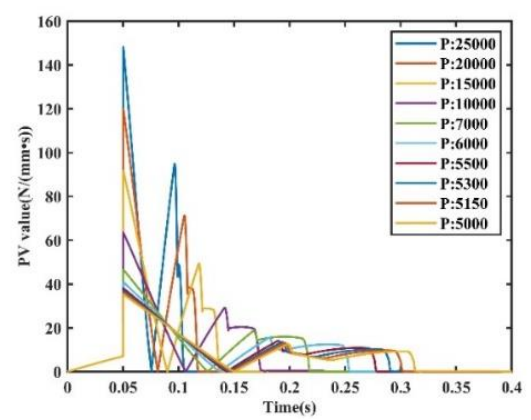

(b) PV value curves for position control with different shifting forces after synchronization

Fig. 19. Synchronizer shift PV value for different shift modes

It can be seen from the figure that the simulated PV value is far less than the allowable value of 15000 $\mathrm{N} /(\mathrm{mm} \bullet \mathrm{s})$. Therefore, it can be seen that the shift simulation in this paper meets the working conditions of the synchronizer.

\subsection{Comparison of the mechanism performance between the PCFRS and the ILRS}

This paper aims to highlight the advantages of the PCFRS from the aspect of mechanical characteristics by comparing the PCFRS and the ILRS, and uses PID control and the same parameters for both synchronizers to achieve a reasonable and comprehensive simulation, so as to obtain the comparison data of the table. If there is a speed difference to realize the shift, the impact torque will be generated. In table 2, the shift data of the ILRS and the PCFRS with similar impact torque are selected, and the data of the PCFRS without speed difference shift data is selected for comparison and explanation.

The ILRS cannot eliminate the speed difference generated during turn-teeth phase, and it will eventually 
generate impact torque. In order to produce a smaller impact torque, a smaller shifting force must be adopted at the expense of shifting time. It can be seen from the table that although the ILRS takes $0.0813 \mathrm{~s}$ to complete the synchronization, when it produces an impact torque similar to that of the PCFRS, the shift time is longer and the impact torque is still greater. It can be seen from the table that the shift performance of the PCFRS in this paper is better than the inertial lock ring synchronizer.

Table 2. Comparison of simulation data between ILRS and PCFRS

\begin{tabular}{cccc}
\hline $\begin{array}{c}\text { Synchronizer } \\
\text { type }\end{array}$ & $\begin{array}{c}\text { synchronization } \\
\text { time/s }\end{array}$ & $\begin{array}{c}\text { Impact } \\
\text { torque } /(\mathrm{N} \cdot \mathrm{m})\end{array}$ & $\begin{array}{c}\text { Shift } \\
\text { time/s }\end{array}$ \\
\hline ILRS & 0.0813 & 69.8256 & 0.2486 \\
PCFRS & 0.0856 & 66.5673 & 0.2031 \\
& & $\begin{array}{c}\text { No speed } \\
\text { difference }\end{array}$ & 0.2406 \\
\hline
\end{tabular}

\section{Conclusion}

A PCFRS, which is suitable for clutchless AMTs of electric vehicles is proposed in this paper, and its mechanical characteristics and rationality of work through simulation analysis is verified. The research results show that the synchronous friction torque can eliminate the speed difference in the turn-teeth phase to achieve no speed difference shifting. Through the verification of evaluation indexes such as specific sliding work and PV value model, it is concluded that the working process of the synchronizer meets the allowable value. The impact torque comparison method is used to measure the characteristics of the shift impact, and the simulation proves that both the simulation step length and the non-flexible components have a great influence on the simulation value of the impact degree.

The shift simulation results of the PCFRS and the ILRS are compared. The wave spring acts on the PCFRS during the shifting process to make the synchronous friction torque always work. Moreover, the PCFRS does not have the turn-ring phase and the lock-teeth phase, which makes the time for shifting without speed difference to be as short as $0.2406 \mathrm{~s}$; When the PCFRS adopts the mode of shifting with a smaller speed difference, the required shifting time is shorter to $0.2031 \mathrm{~s}$. Compared with the ILRS that shifting time is $0.2486 \mathrm{~s}$, the impact torque is smaller as well as the time required is shorter.

In addition, the mechanism studied in this paper still has problems to be solved, such as not optimizing the springs in the gear shift phase; the structure of the PCFRS has been determined, but the corresponding size is only preliminary determined in combination with the structural characteristics. In the next st ep, the relationship between the characteristics of the wave spring and the gear shifting force will be further analyzed, and the size of the mechanism will be optimized.

\section{Declaration}

\section{Funding}

Supported by the National Natural Science Foundation of China (Grant No. 51775478) and Natural Science Foundation of Hebei Province (Grant No: E2020203078).

\section{Availability of data and materials}

The datasets supporting the conclusions of this article are included within the article.

\section{Authors' contributions}

The authors' contributions are as follows: wrote the manuscript; simulation analyses and compared to verify.

\section{Competing interests}

The authors declare no competing financial interests.

\section{Consent for publication}

Not applicable

\section{Ethics approval and consent to participate \\ Not applicable}

\section{References}

[1] C Tseng, C Yu. Advanced shifting control of synchronizer mechanisms for clutchless automatic manual transmission in an electric vehicle. Mechanism \& Machine Theory, 2015, 84:37-56.

[2] P D Walker, S A Rahman, Z Bo, et al. Modelling, simulations, and optimisation of electric vehicles for analysis of transmission ratio selection. Advances in Mechanical Engineering, 2013, (2013-12-1), 2013, 5(2013):1-7.

[3] H X Chen, G Y Tian. Modeling and simulation of shift process of electric motor-transmission system. Journal of Tsinghua University (Science and Technology), 2016, 56(02):144-151.

[4] H He, Z Liu, L Zhu, et al. Dynamic coordinated shifting control of automated mechanical transmissions without a clutch in a plug-in hybrid electric vehicle. Energies, 2012, 5(8):3094-3109.

[5] Z Ma, S Cheng, C Q Wang. Analysis of shift performance of synchronizer of mechanical transmission. New Technology \& New Products of China, 2018, 16:37-38.

[6] B Huang, S Wu, X Fu, et al. Research on the shift process of electrically driven mechanical automatic transmission. Automobile Technology, 2015, 7:18-23.

[7] C Yu, C Tseng. Research on gear-change control technology for the clutchless automatic-manual transmission of an electric vehicle. Proceedings of the Institution of Mechanical Engineers Part D Journal of Automobile Engineering, 2013, 227(10):1446-1458.

[8] J Li, F Luo, Y Luo, et al. Sensitivity analysis on a synchronization mechanism for manual transmission gearbox, SAE Technical Papers. 2014, 1:1768.

[9] X Y Wang, L Li, K He, et al. Position and force switching control for gear engagement of automated manual transmission gear-shift process. Journal of Dynamic Systems Measurement \& Control, 2018, 140(8):4039184. 
[10] W L Xu, W Zhao, B Su, et al. Investigation of manual transmission synchronizer failure mechanism induced by interface material/lubricant combinations. Wear, 2015, 328-329:436-442.

[11] D T Qin, M Y Yao, S J Chen, et al. Shifting process control for two-speed automated mechanical transmission of pure electric vehicles. International Journal of Precision Engineering and Manufacturing, 2016, 17(5):623-629.

[12] S S Lin, S Q Chang, B Li. Gearshift control system development for direct-drive automated manual transmission based on a novel electromagnetic actuator. Mechatronics, 2014, 24(8):1214-1222.

[13] L P Zhang, L Q Yang, X B Guo, et al. Stage-by-phase multivariable combination control for centralized and distributed drive modes switching of electric vehicles. Mechanism and Machine Theory, 2020, 147:103752.

[14] Y Zhang, H Zhao, M M Qiu, et al. Model-based control of synchronizer shifting process for trajectory tracking control. International Journal of Automotive Technology, 2020, 21(4):943-952.

[15] G G You, Y Cheng, J N Li. Working process analysis and parameter optimization of lock ring synchronizer. Journal of Mechanical Transmission, 2016, 2:73-76.

[16] Q S Zhao, C M Chu, H C Tang, et al. Research on the influence of synchronizing ring cone parameters on synchronizer performance. Journal of Mechanical Transmission, 2014, 38(01):107-111.

[17] W L Xu, W Zhao, X Zhang, et al. Analysis on failure process and failure mechanism of transmission synchronizer. Journal of Mechanical Engineering, 2014, 50(14):69-74.

[18] C H Wang, Z W Guo. Synchronizer multi-objective parameter optimization based on improved particle swarm algorithm. Journal of Mechanical Strength, 2019, 2:356-362.

[19] S T Razzacki. Synchronizer design: a mathematical and dimensional treatise. SAE Transactions, 2004, 1:1230.

[20] L Li, K He, X Wang, et al. Sensor fault-tolerant control for gear-shifting engaging process of automated manual transmission. Mechanical Systems \& Signal Processing, 2018, 99:790-804.

[21] Y F Zeng, H Chen, L J Wang, et al. Modeling and control of the shifting process of a motor-transmission system without a synchronizer. Journal of Tsinghua University (Natural Science Edition), 2020, 60(11):101-108.

[22] F Tian, L J Wang, L Q Sui, et al. Active gear alignment control in the shift process of electric vehicle synchronizerless transmission. Journal of Tsinghua University (Science and Technology), 2020, 60(02):4-11.

[23] R Heath. Zeroshift's transmission technology. Atzautotechnology, 2008, 8(11-12):44-49.

[24] W Mo, P D Walker, N Zhang. Dynamic analysis and control for an electric vehicle with harpoon-shift synchronizer. Mechanism and Machine Theory, 2019, 133:750-766.

[25] W Mo, P Walker, Y Fang, et al. A novel shift control concept for multi-speed electric vehicles. Mechanical Systems and Signal Processing, 2018, 112:171-193.

[26] M Mousavi, A Pakniyat, T Wang, et al. Seamless dual brake transmission for electric vehicles: Design, control and experiment. Mechanism and Machine Theory, 2015, 94:96-118.

[27] Y Tian, J Ruan, N Zhang, et al. Modelling and control of a novel two-speed transmission for electric vehicles. Mechanism \& Machine Theory, 2018, 127:13-32.

[28] B Z Gao, Q Liang, Y Xiang, et al. Gear ratio optimization and shift control of 2-speed I-AMT in electric vehicle. Mechanical Systems and Signal Processing, 2015, 50-51:615-631.

[29] L P Zhang, D J Gu, B N Qi, et al. Variable-mode impact suppression method for electric vehicle dual-mode coupling drive system, Journal of Mechanical Engineering, 2018, 54(08):165-176.

[30] F K Omar, K A Moustafa, S Emam. Mathematical modeling of gearbox including defects with experimental verification. Journal of Vibration \& Control, 2012, 18(9):1310-1321.

[31] S Zhou, Y B Cao. Research on AMT shift schedule based on improving shift quality. Machinery Design \& Manufacture, 2008, 7:138-140

[32] G Hui, Y Hao. Study on the influence of shift parameter on the synchronizing process of lock ring synchronizer. Journal of Mechanical Transmission, 2014, 38(12):115-118.

[33] H Naunheimer. Automotive transmission, Springer-Verlag Berlin Heidelberg, 2011.

[34] L T Cao. Wear analysis and optimization design of synchronizer of two-speed transmission in pure electric vehicle, Hefei University of Technology, 2016.

\section{Biographical notes}

Li-Peng Zhang, born in 1979, is currently a professor at Yanshan University, China. He received his PhD degree from Beijing Institute of Technology, China, in 2011. His research interests include intelligent vehicle dynamics and control, new energy vehicle compound transmission, driver awareness and driving assistance.

Tel: +86-18533537646; E-mail: evzlp@ysu.edu.cn

Yun-Ao Peng, born in 1996, is currently a master candidate at Hebei Key Laboratory of Special Delivery Equipment, Yanshan University, China.

E-mail: 2667940666@qq.com

Hao-Jie Yang, born in 1998, is currently a master candidate at Hebei Key Laboratory of Special Delivery Equipment, Yanshan University, China.

E-mail: 798056973@qq.com 
Figures

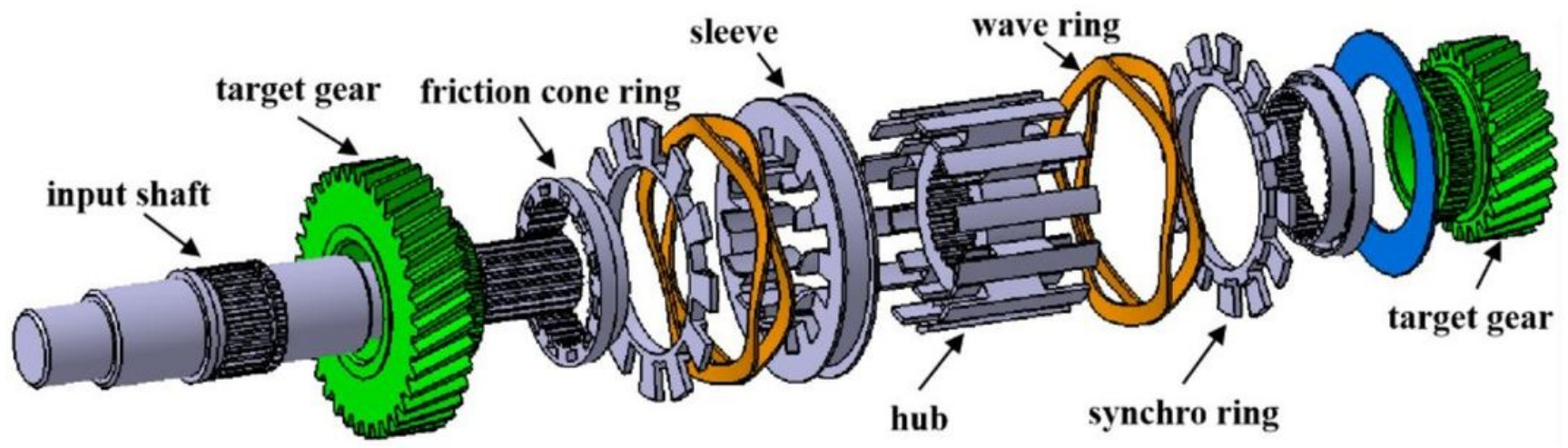

Figure 1

The structure and assembly relationship of PCFRS

\section{pressure-controllable friction ring synchronizer}

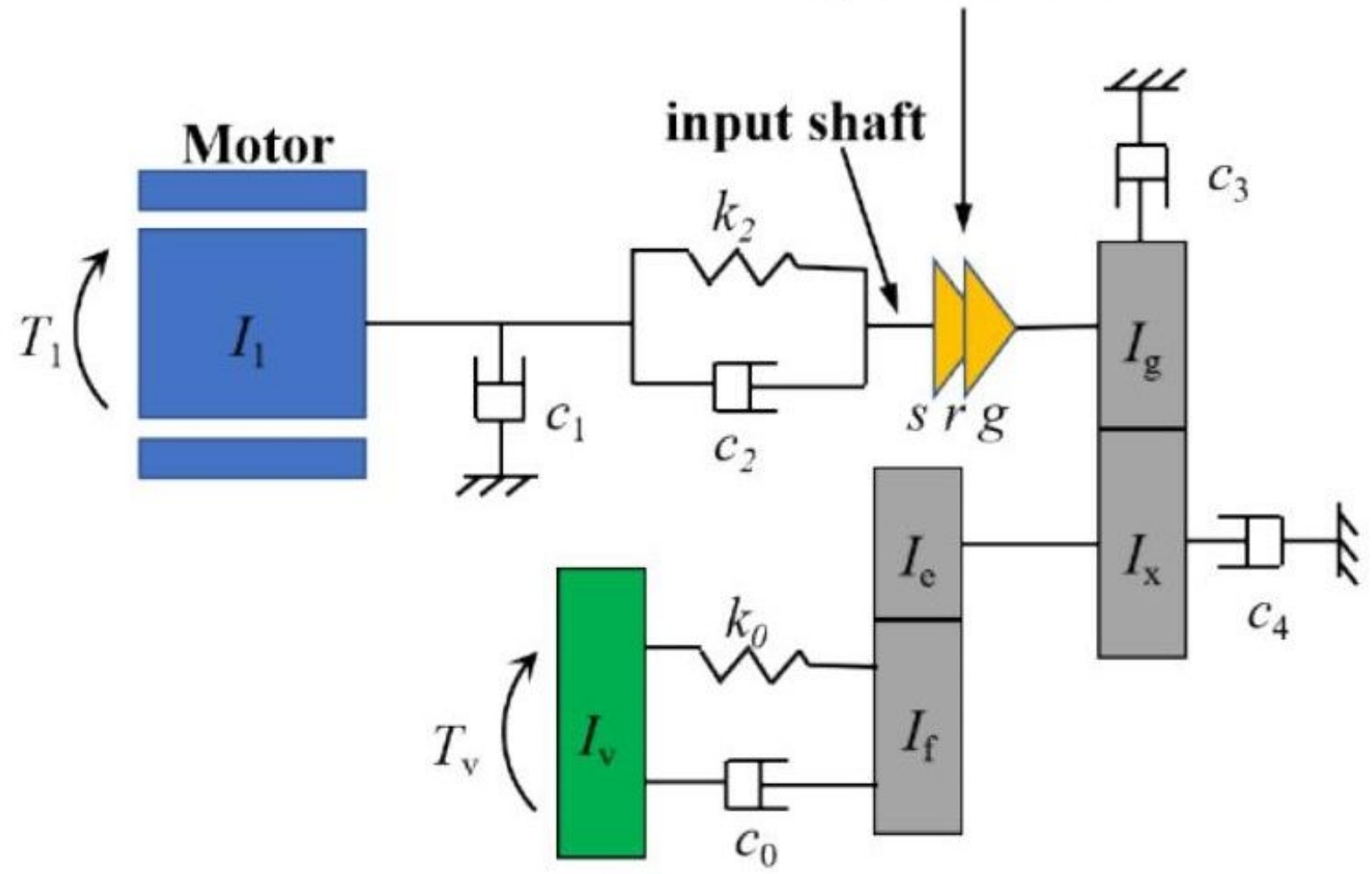

Figure 2 


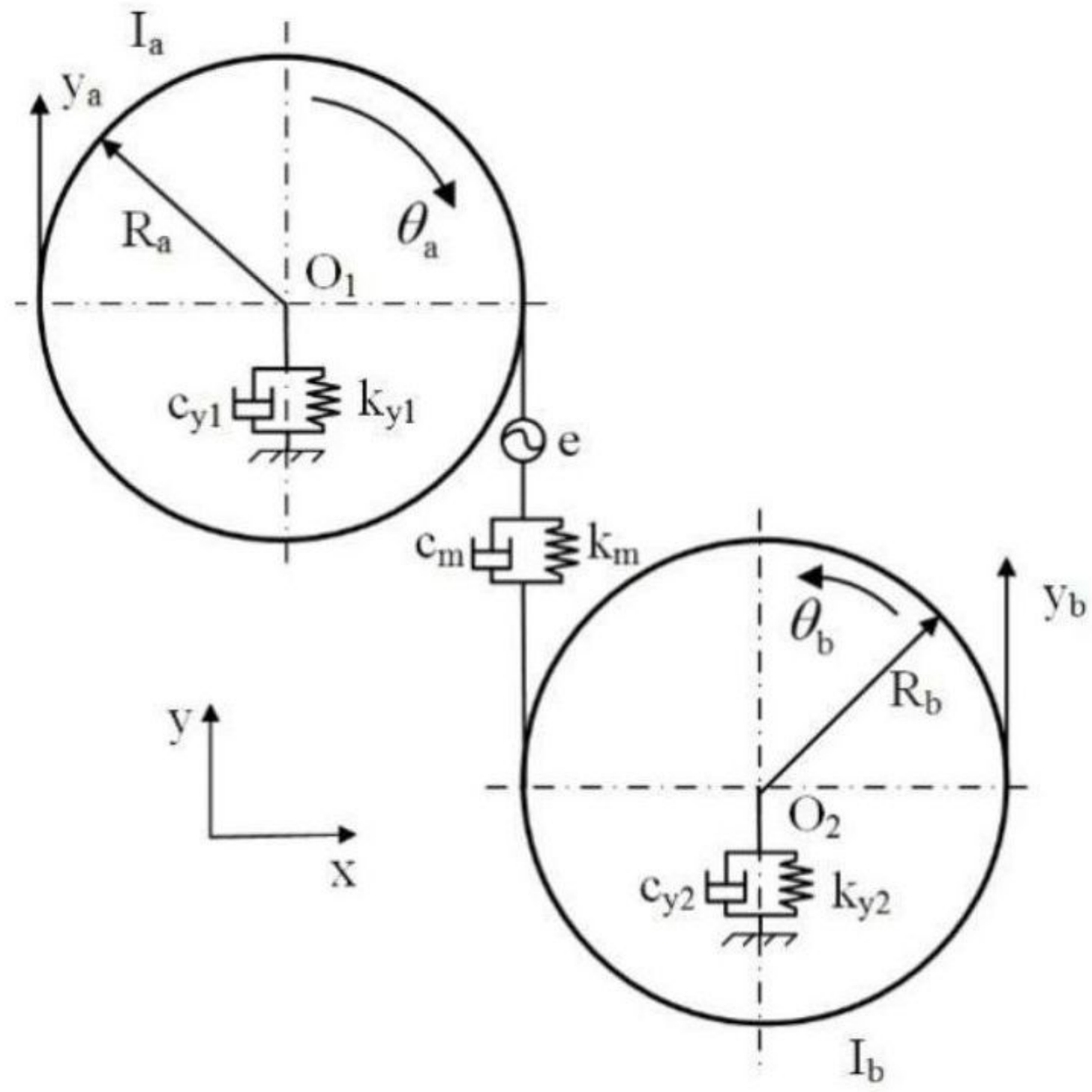

Figure 3

Non-linear dynamics model of gear meshing 


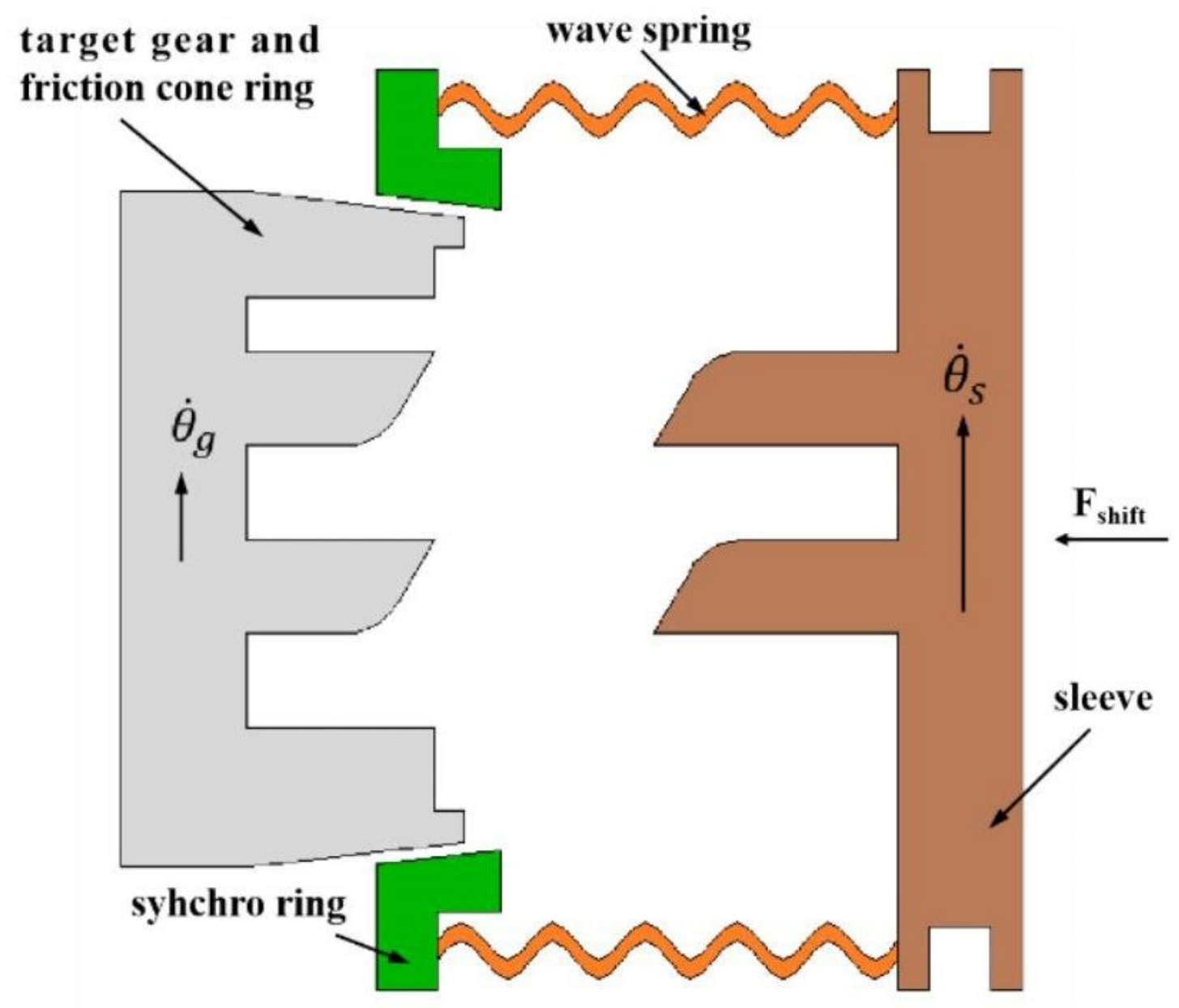

Figure 4

Schematic diagram of the structure of the sleeve in the middle position of the synchronizer 

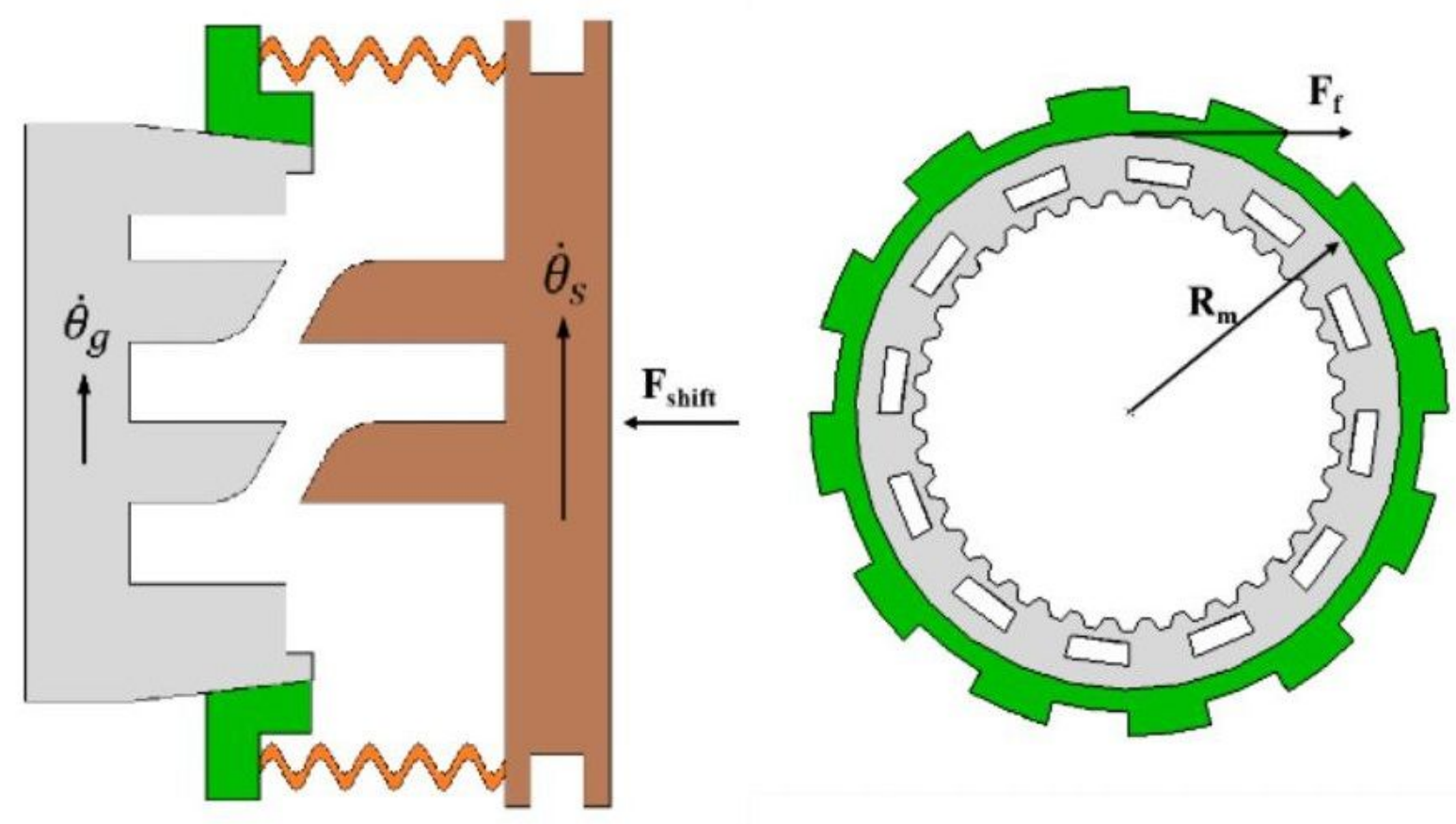

Figure 5

Schematic diagram of the synchronization process of the synchro ring and the friction cone ring contacting 


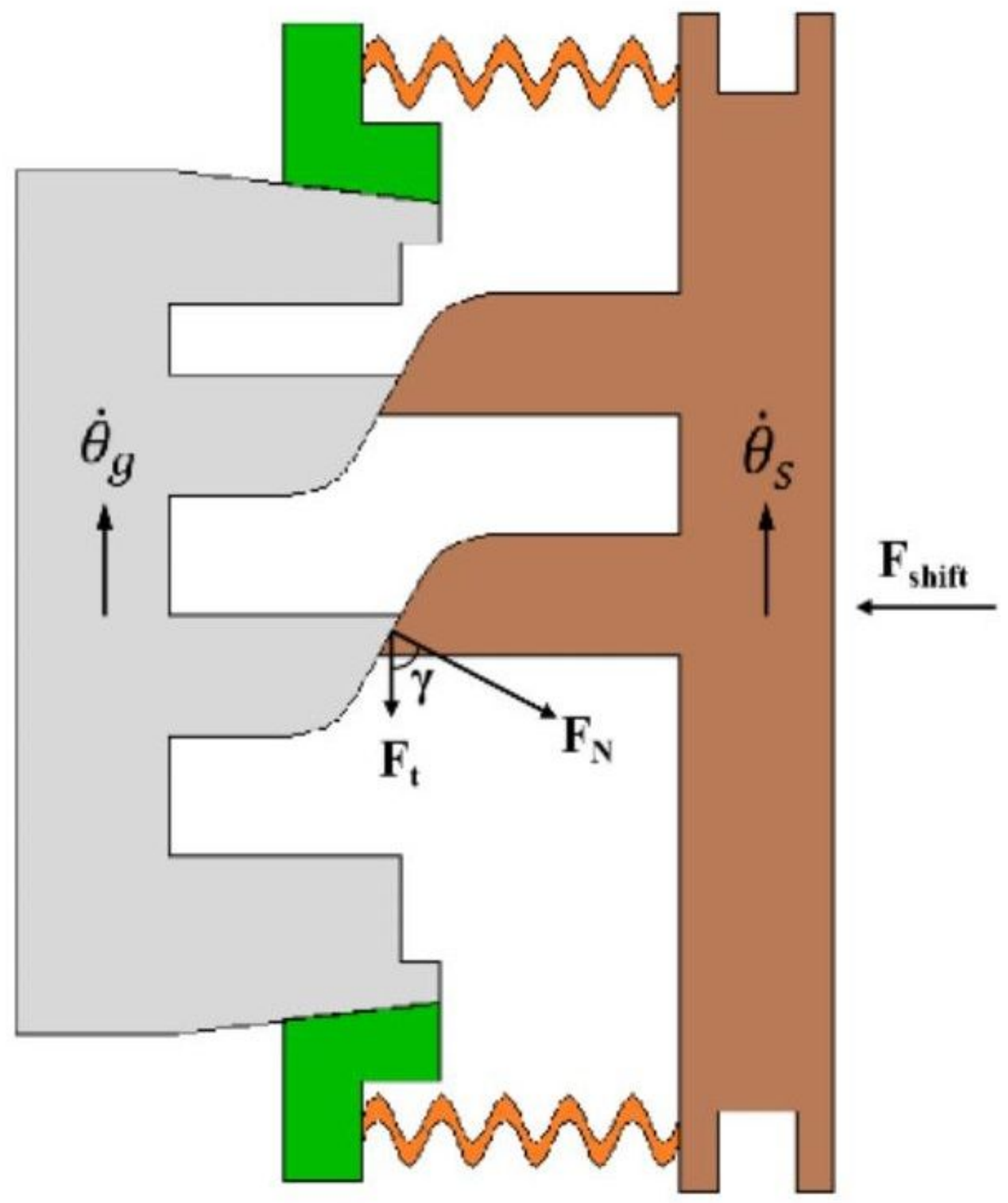

Figure 6

Schematic diagram of the structure of the sleeve and the friction cone ring contacting 


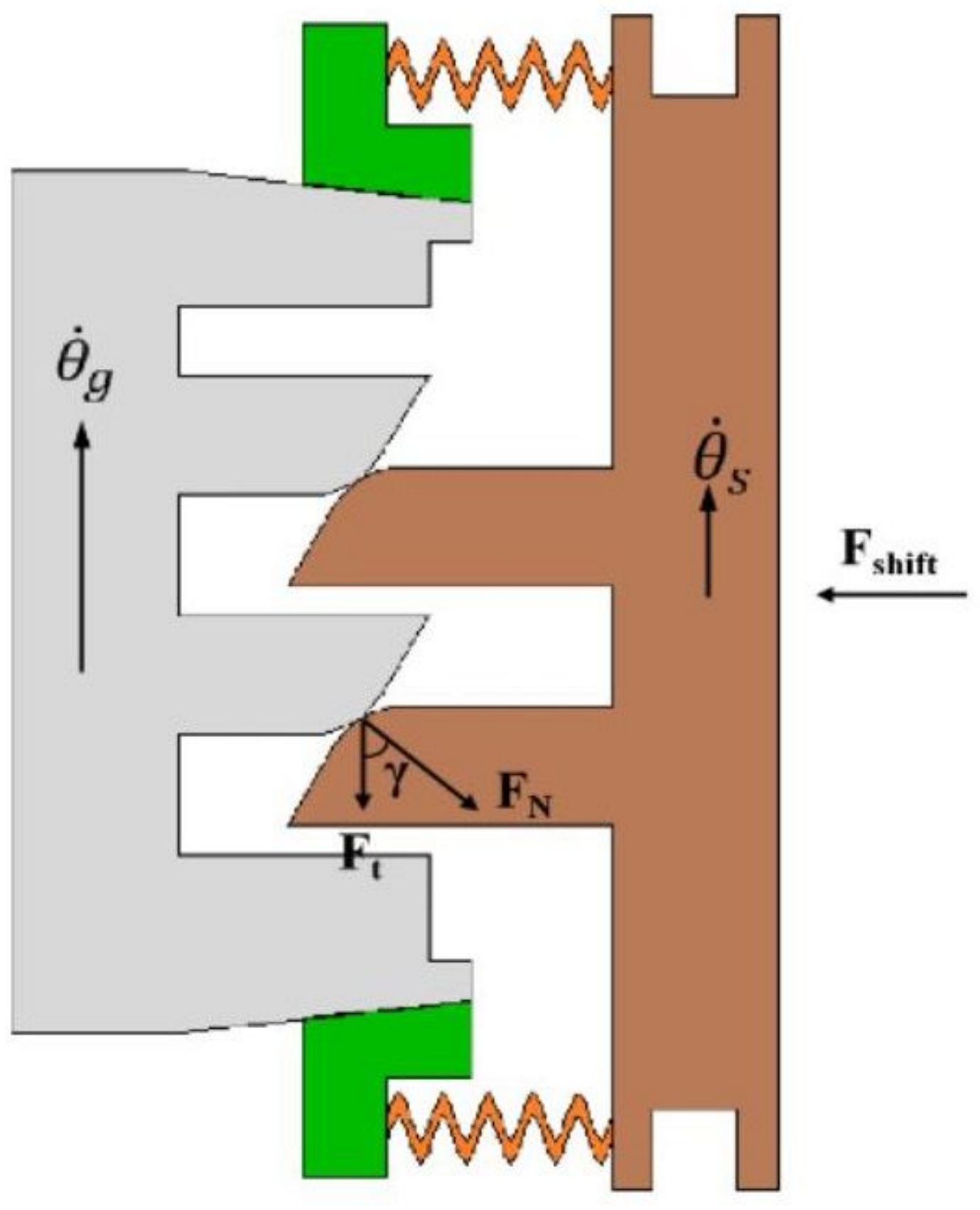

Figure 7

Schematic diagram of the turning teeth after the sleeve teeth and the friction cone ring teeth contacting 


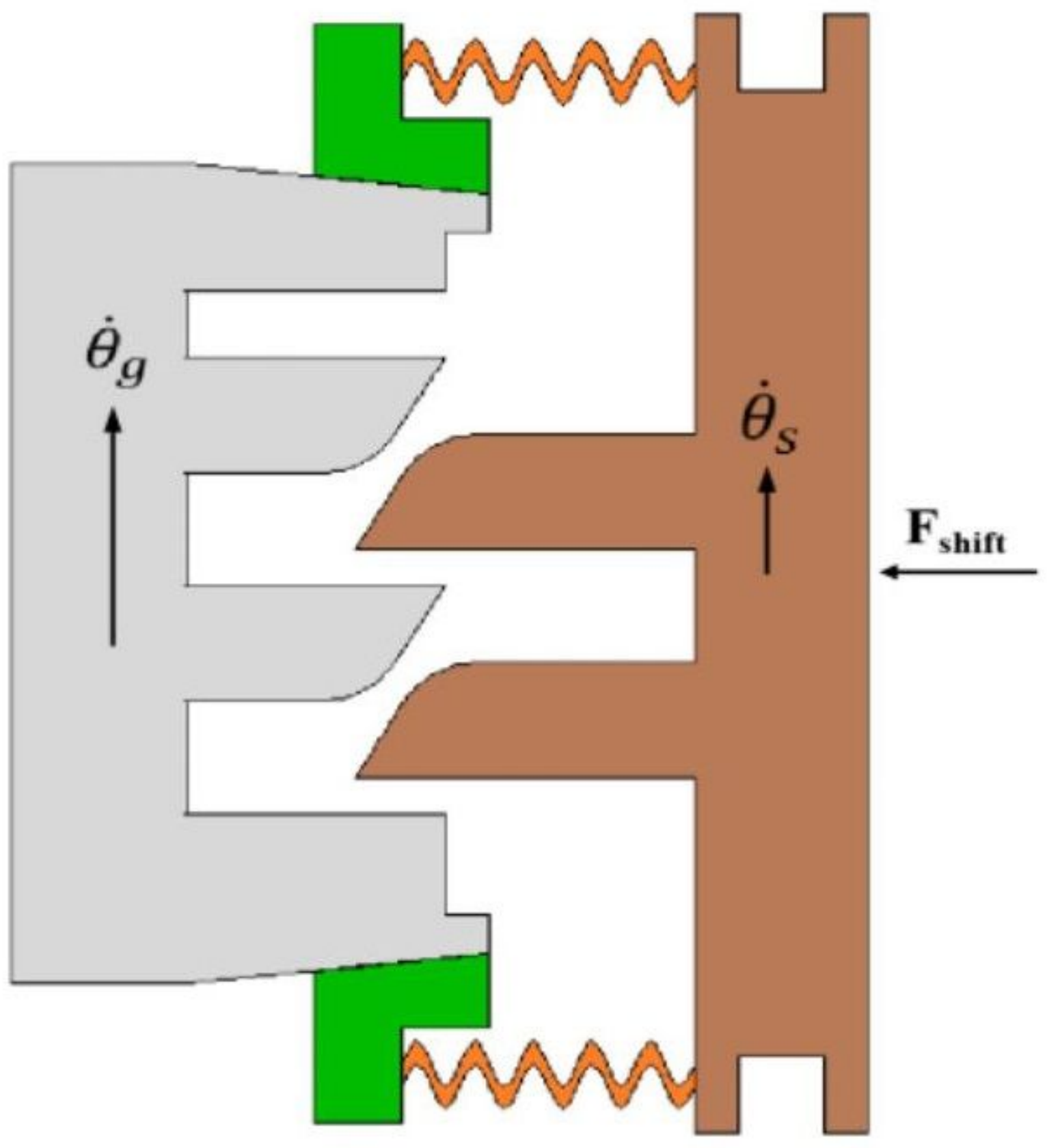

Figure 8

Schematic diagram of the structure of using the speed difference generated by the turn-teeth to complete the shifting 


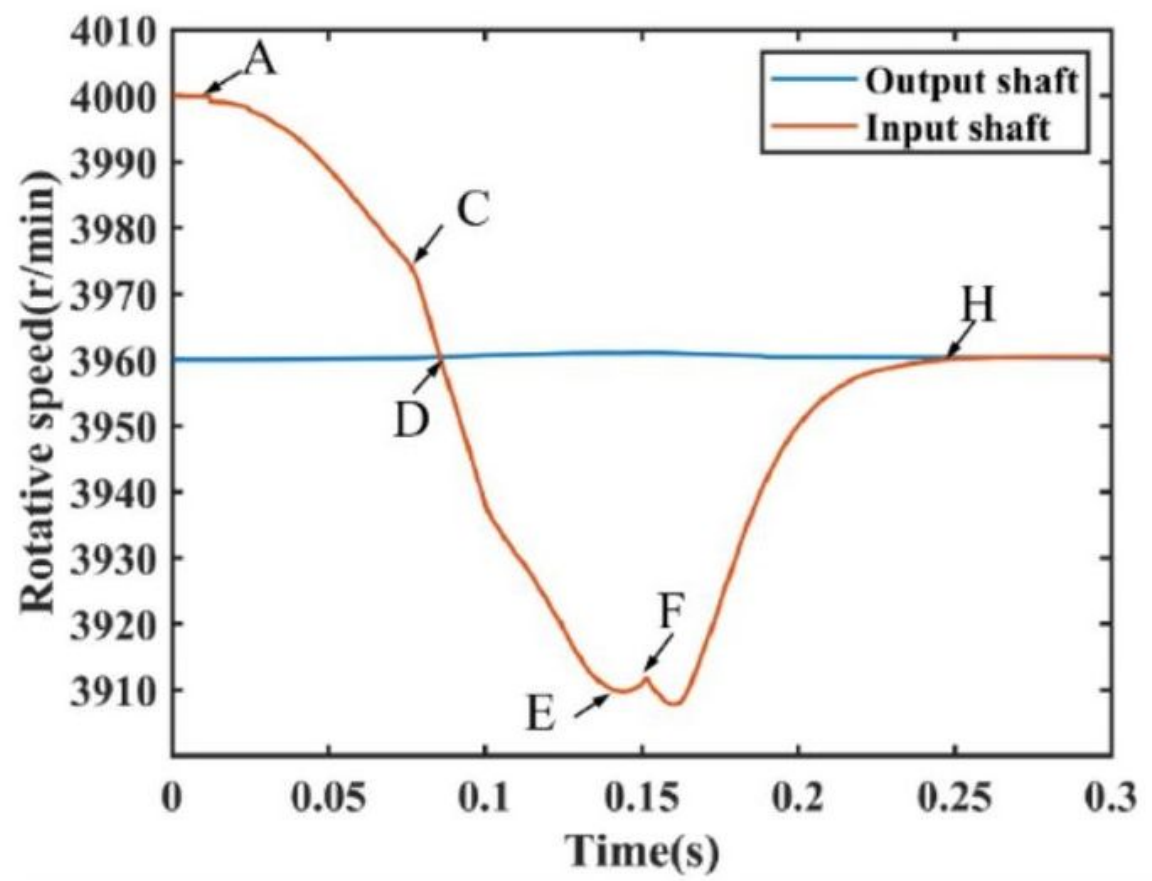

(a) Speed curves of the input shaft and the output shaft

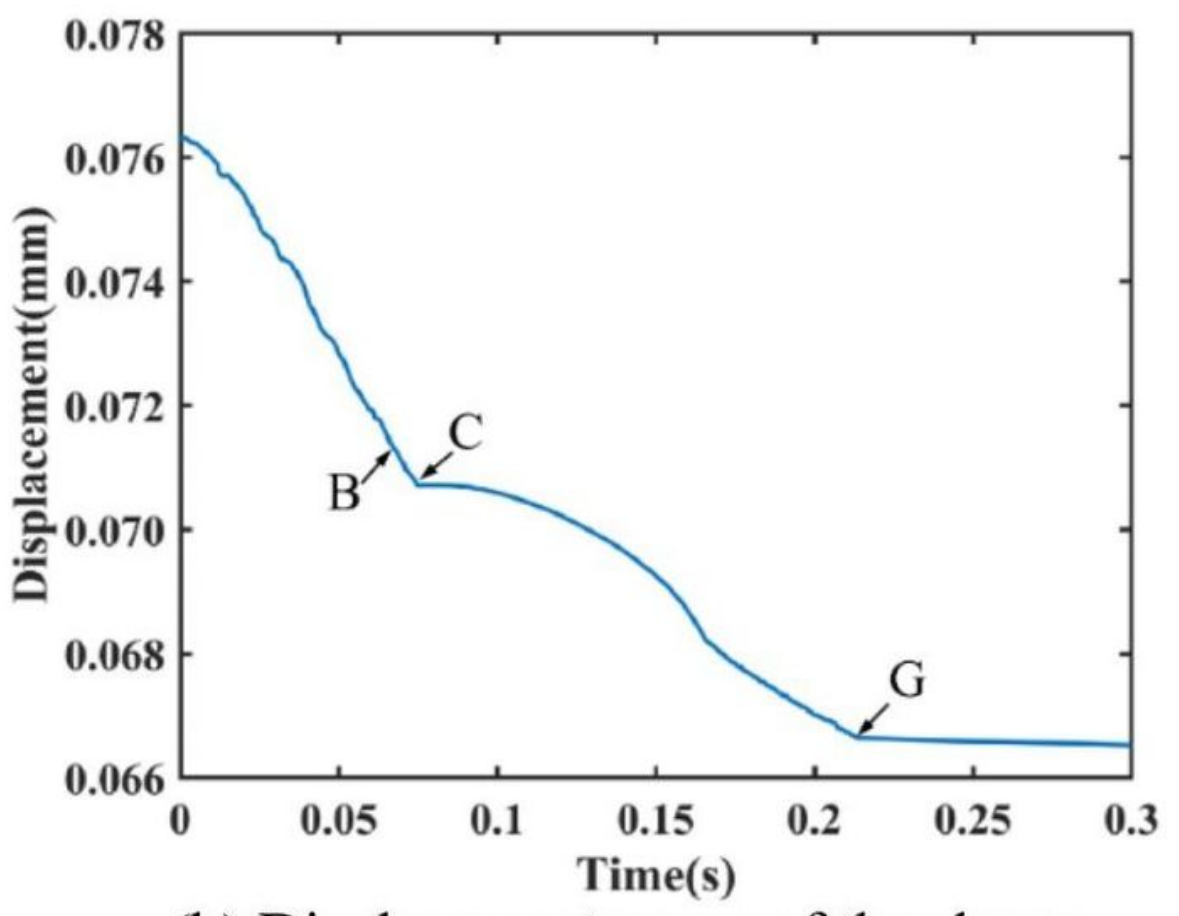

(b) Displacement curve of the sleeve

Figure 9

Transmission movement state during the shift 


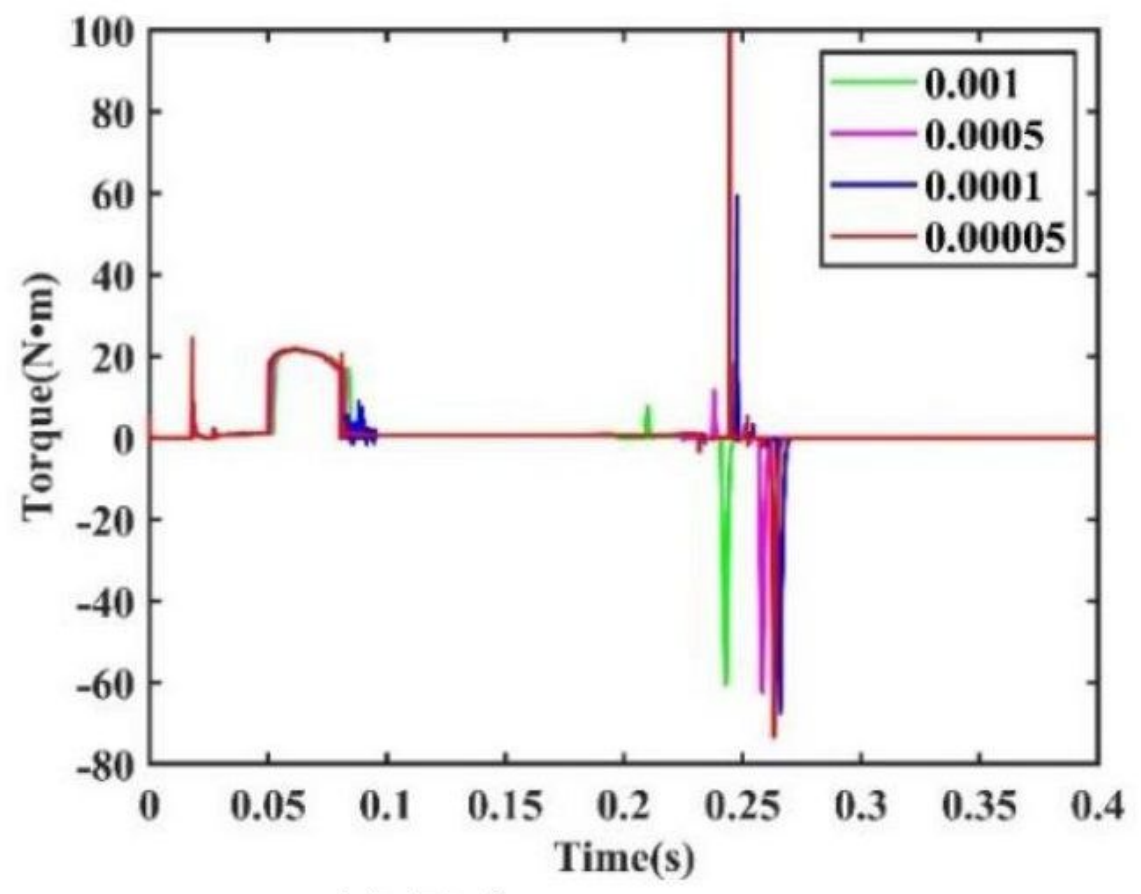

(a) Drive torque curves

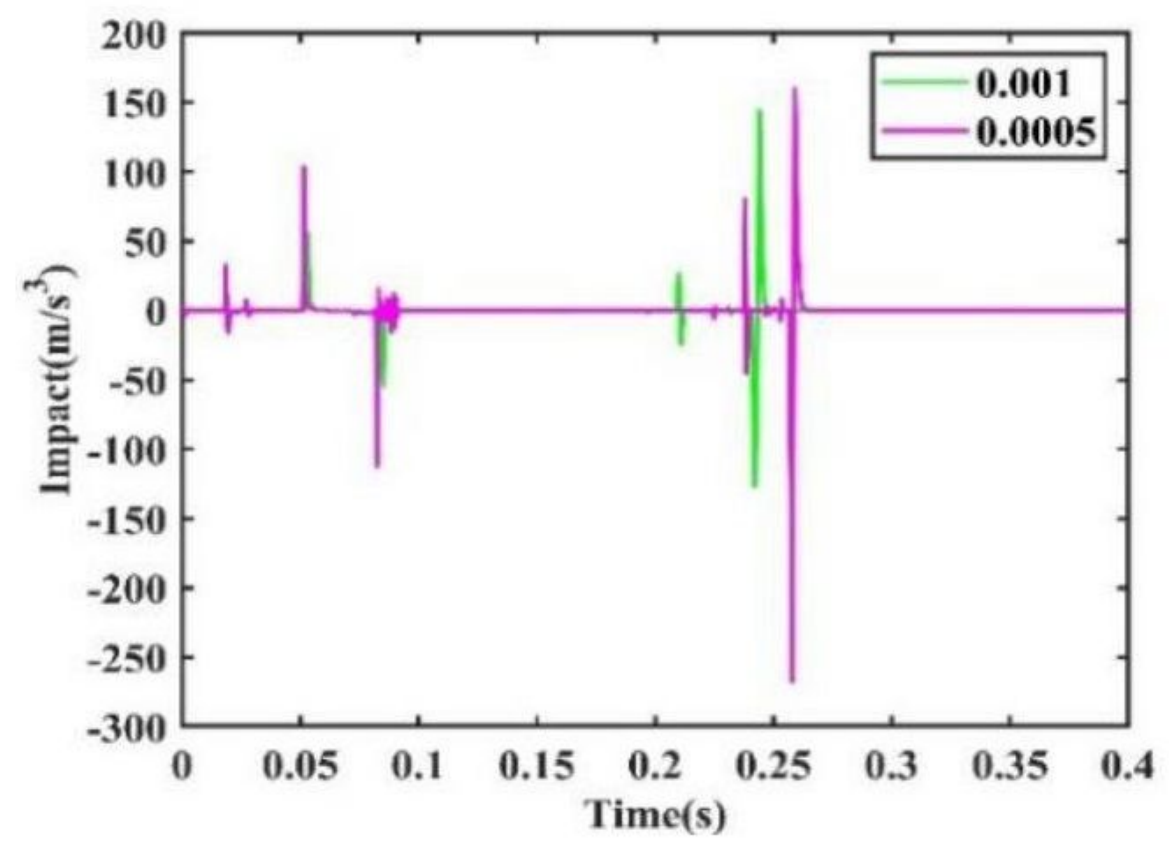

(b) Impact curves

Figure 10

Curves of the drive torque and the impact obtained by simulation of different simulation steps 


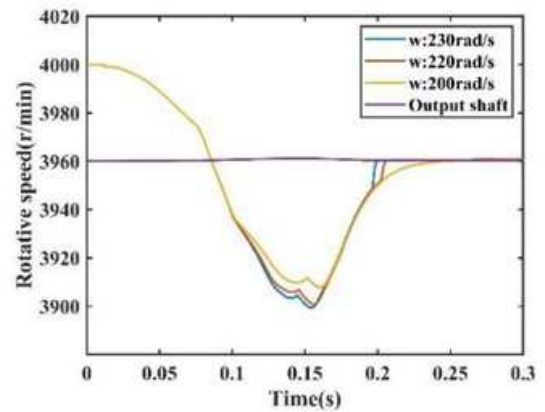

(a) The speed curves of the input shaft and the output shaft with different moving speeds of the sleeve

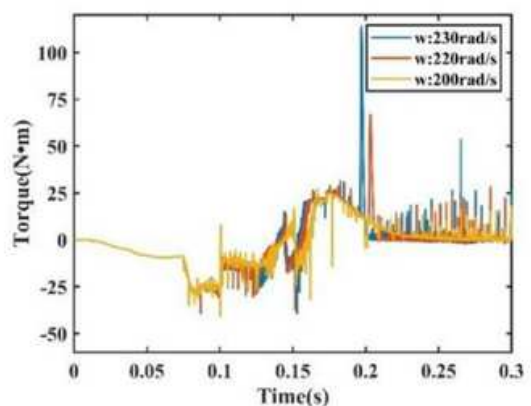

(b) Impact torques of different shift effects and target gear output speed

\section{Figure 11}

Shift effect of PCFRS at different sleeve moving speeds 


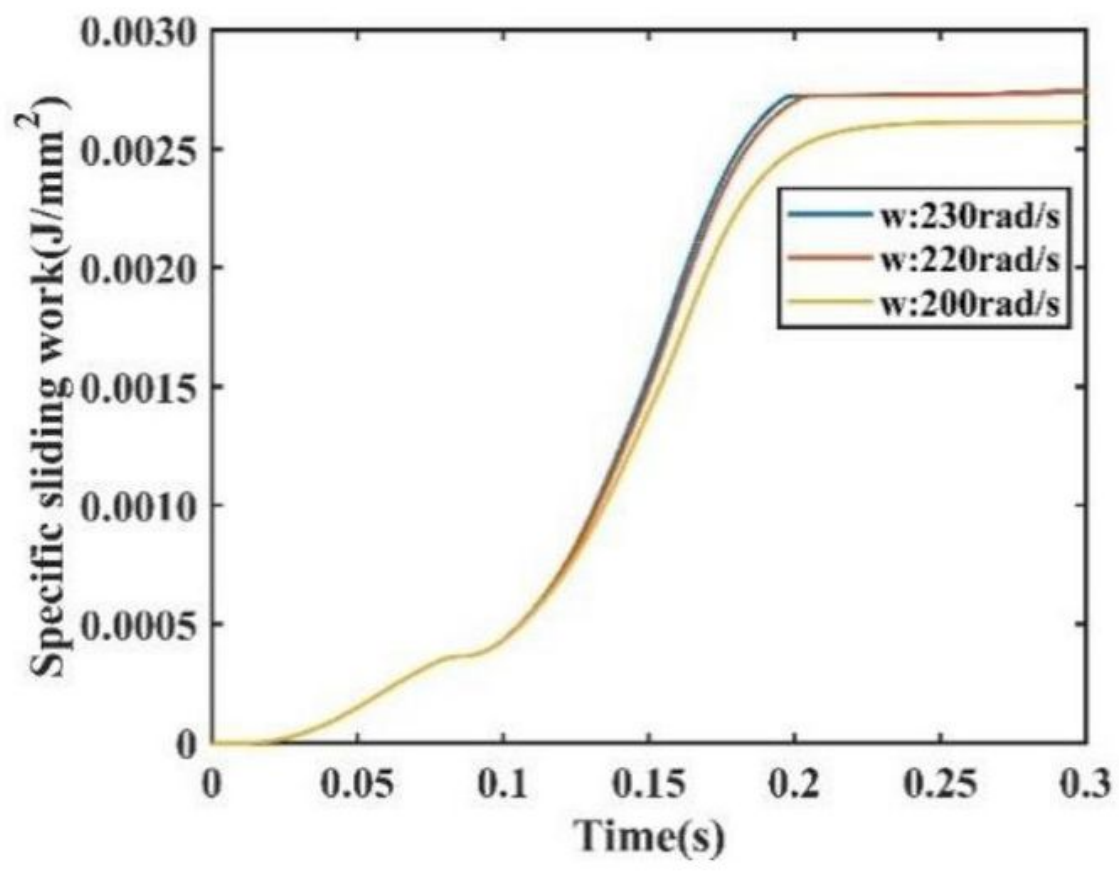

(a) Specific sliding work curves

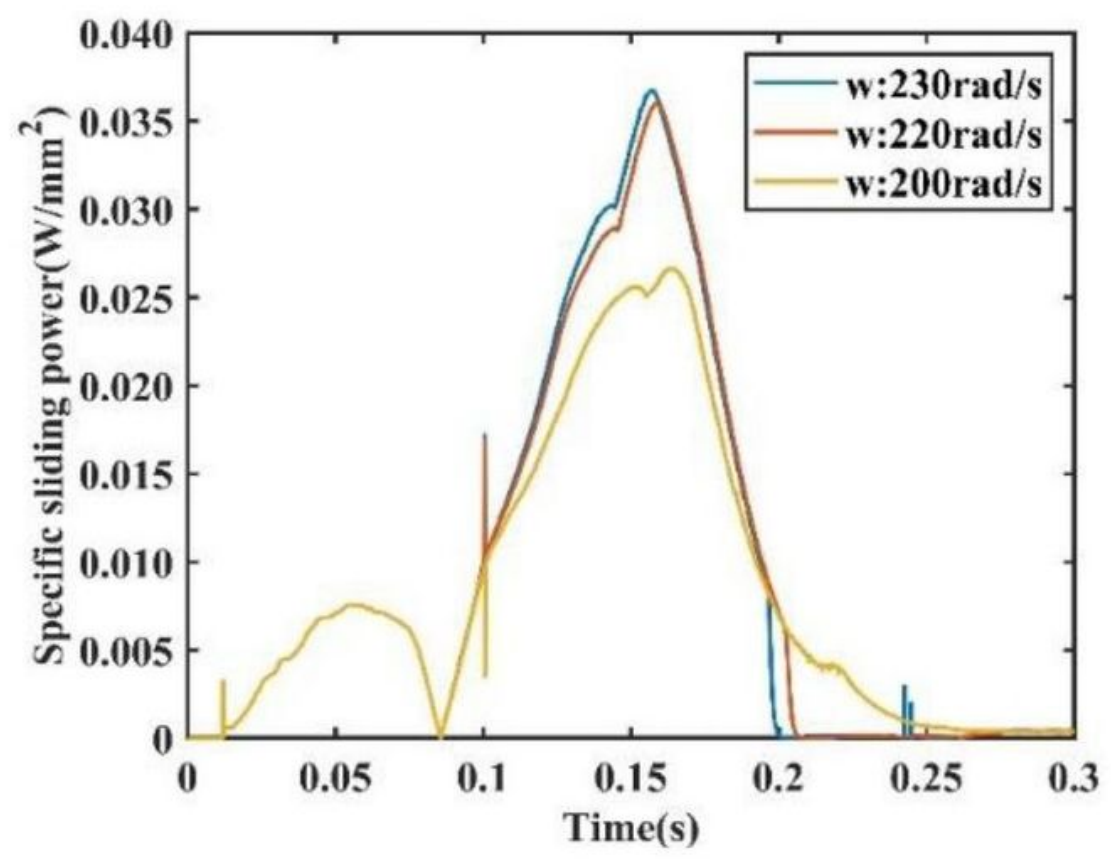

(b) Specific sliding power curves

Figure 12

The specific sliding work and specific sliding power of the PCFRS under different shift speeds 


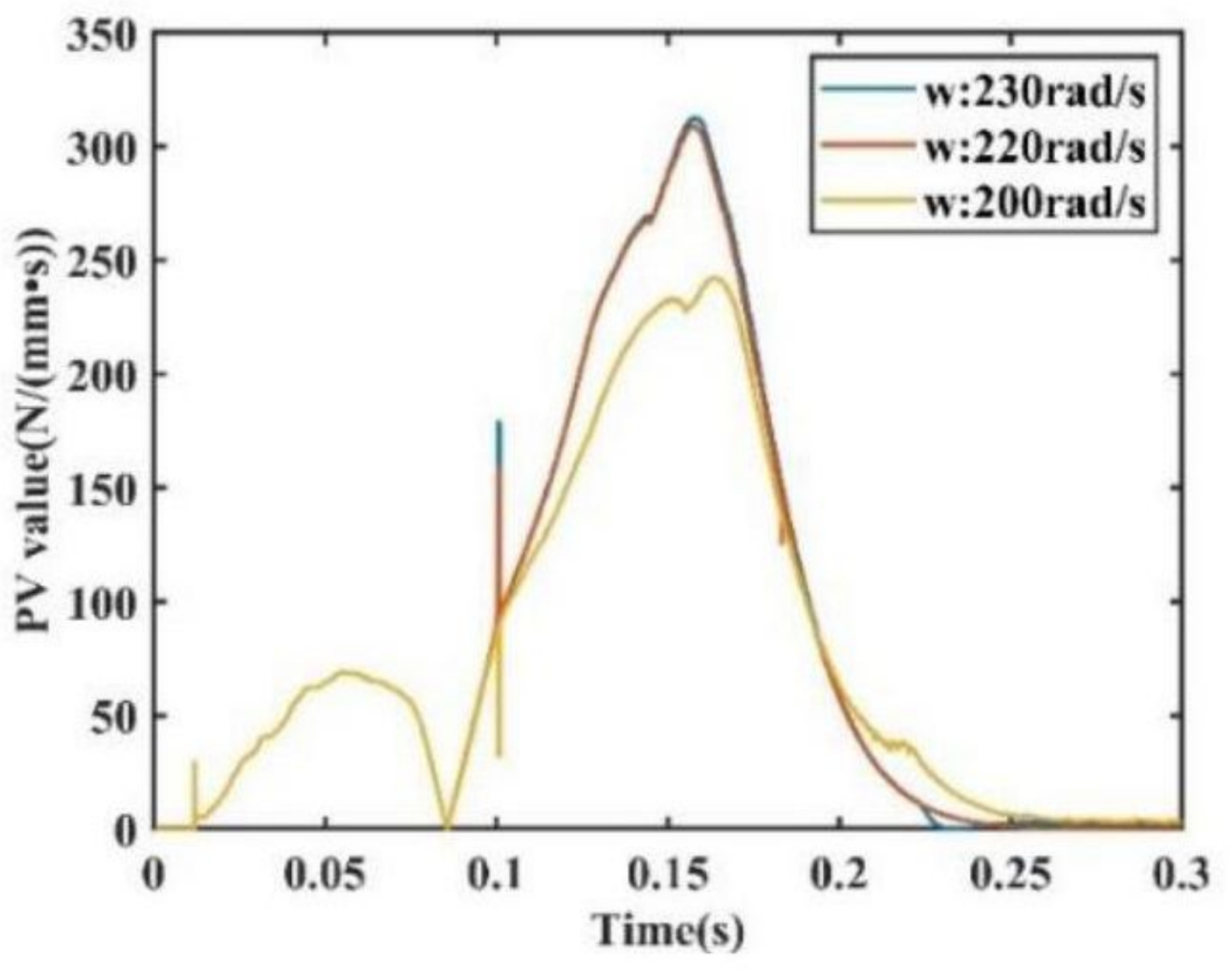

Figure 13

PV value curves of the PCFRS 


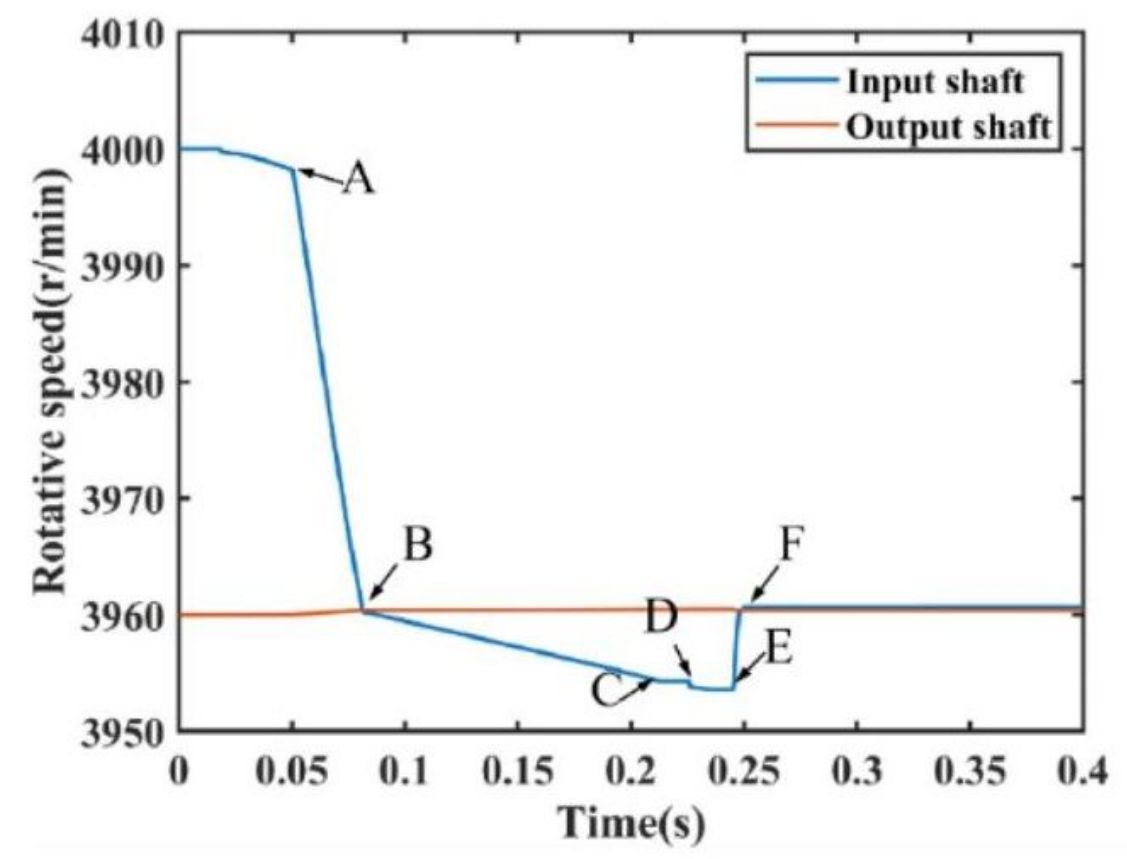

(a) Speed curves of the input shaft and the output shaft

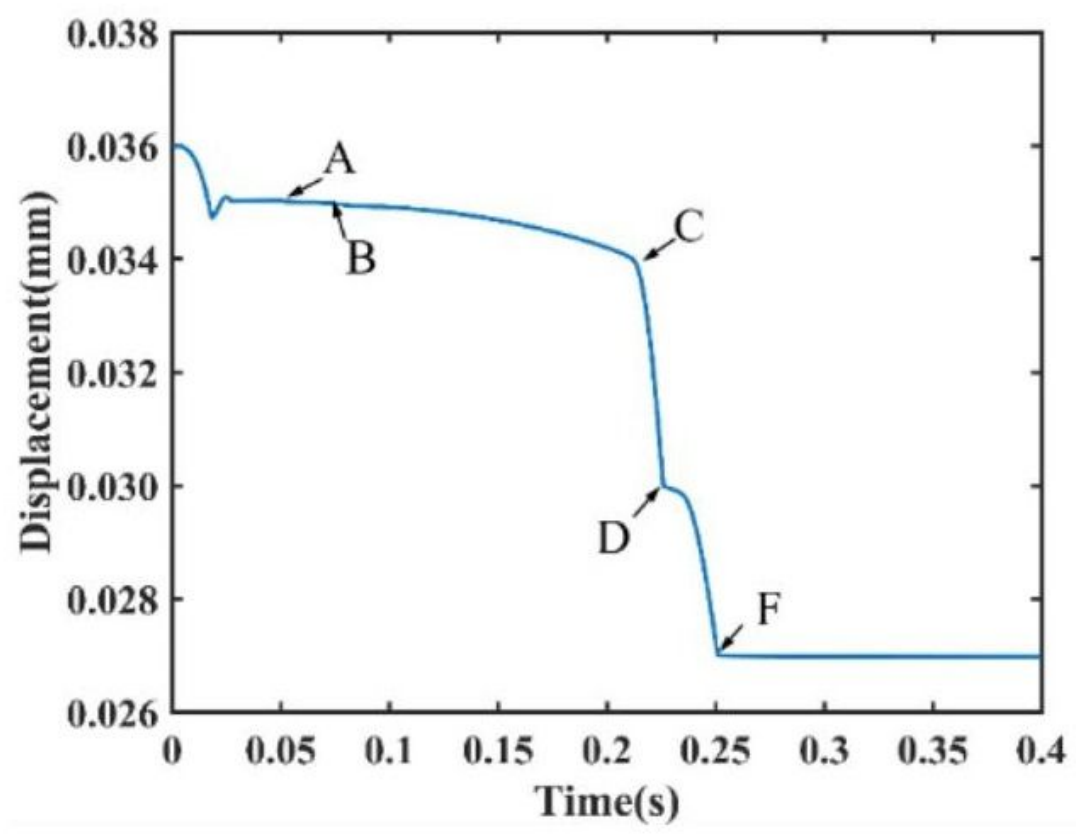

(b) Displacement curves of the sleeve

Figure 14

Transmission movement state during shift 


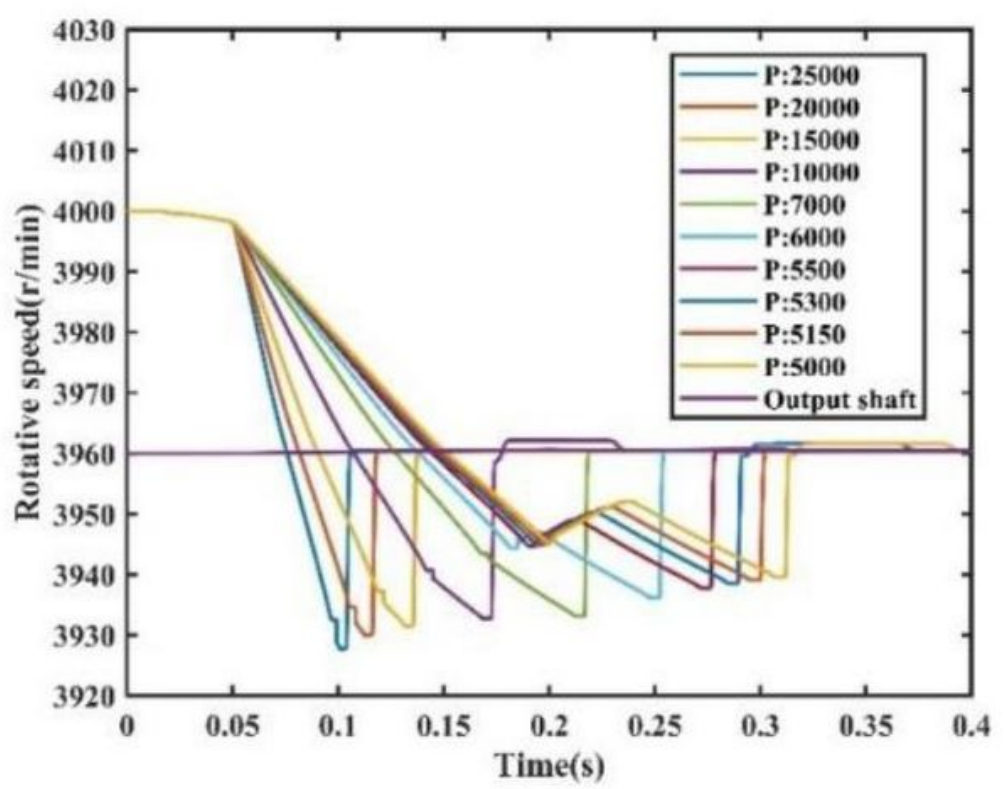

(a) The speeds relationship between the input shaft and the output shaft

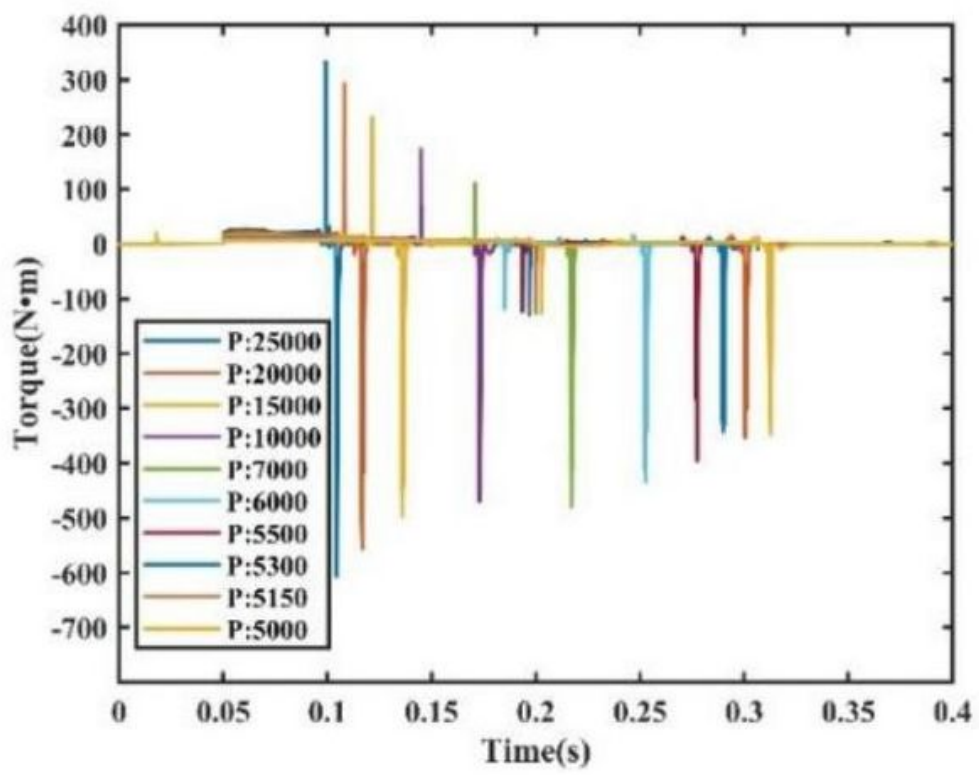

(b) Impact torque curves of different shift modes

Figure 15

Shift effect achieved by ILRS with single position control 


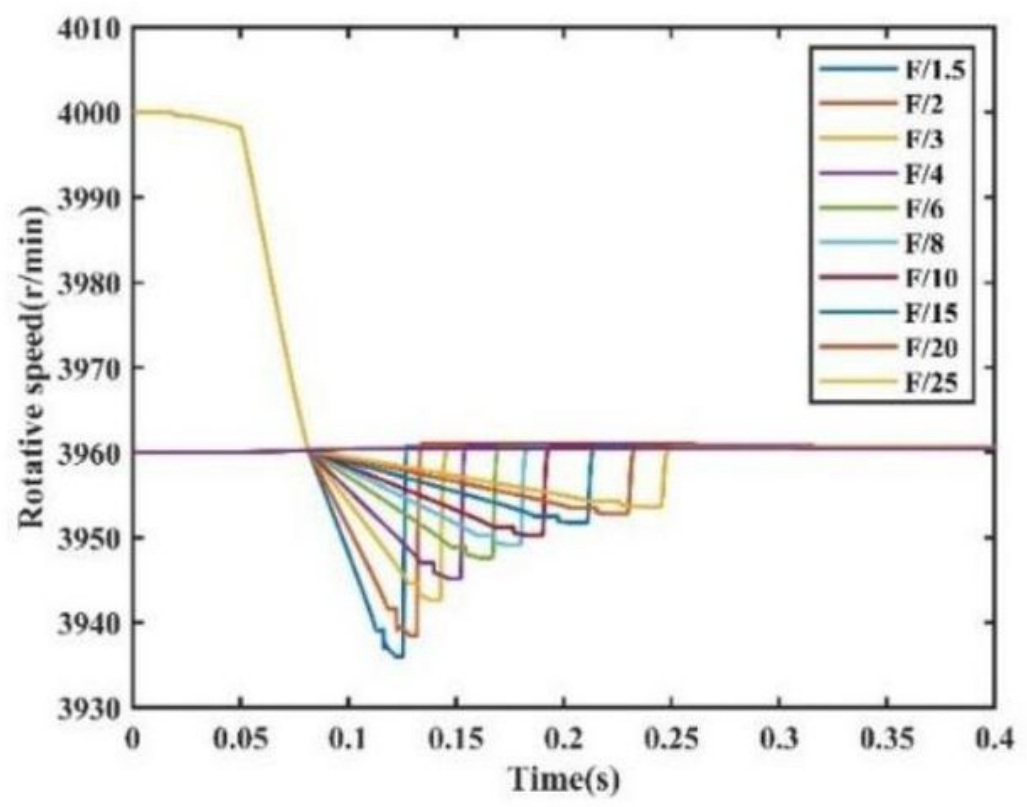

(a) The speeds relationship between the input shaft and the output shaft

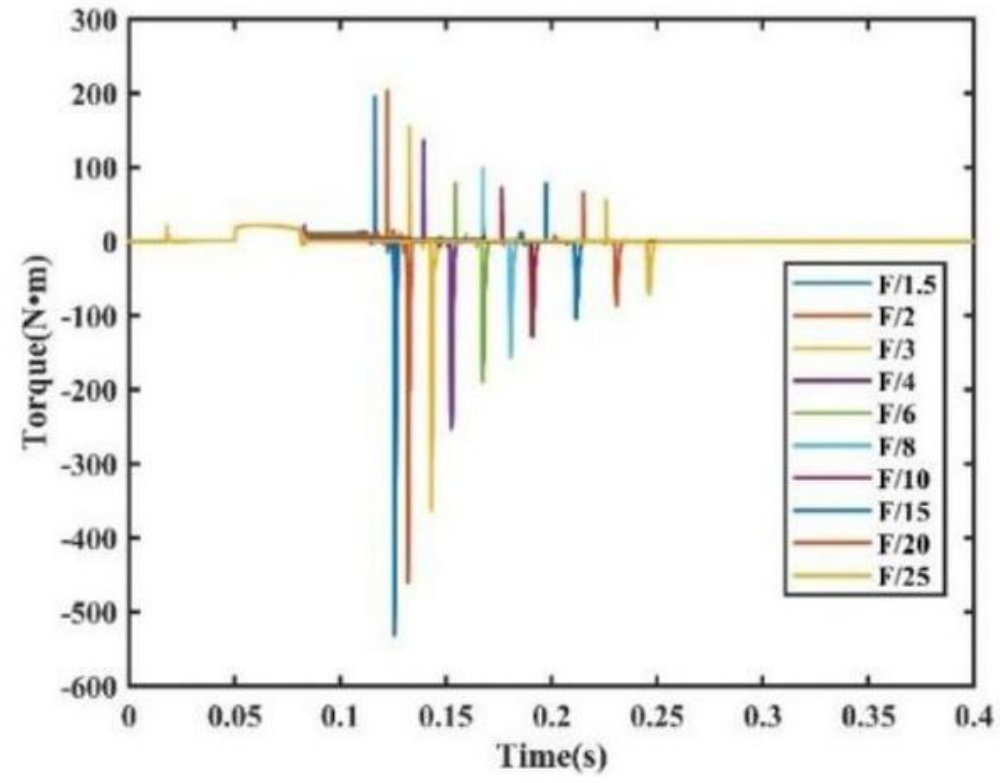

(b) Impact torque curves of different shift modes

Figure 16

Shifting effect of ILRS adopts different shifting force after synchronization 


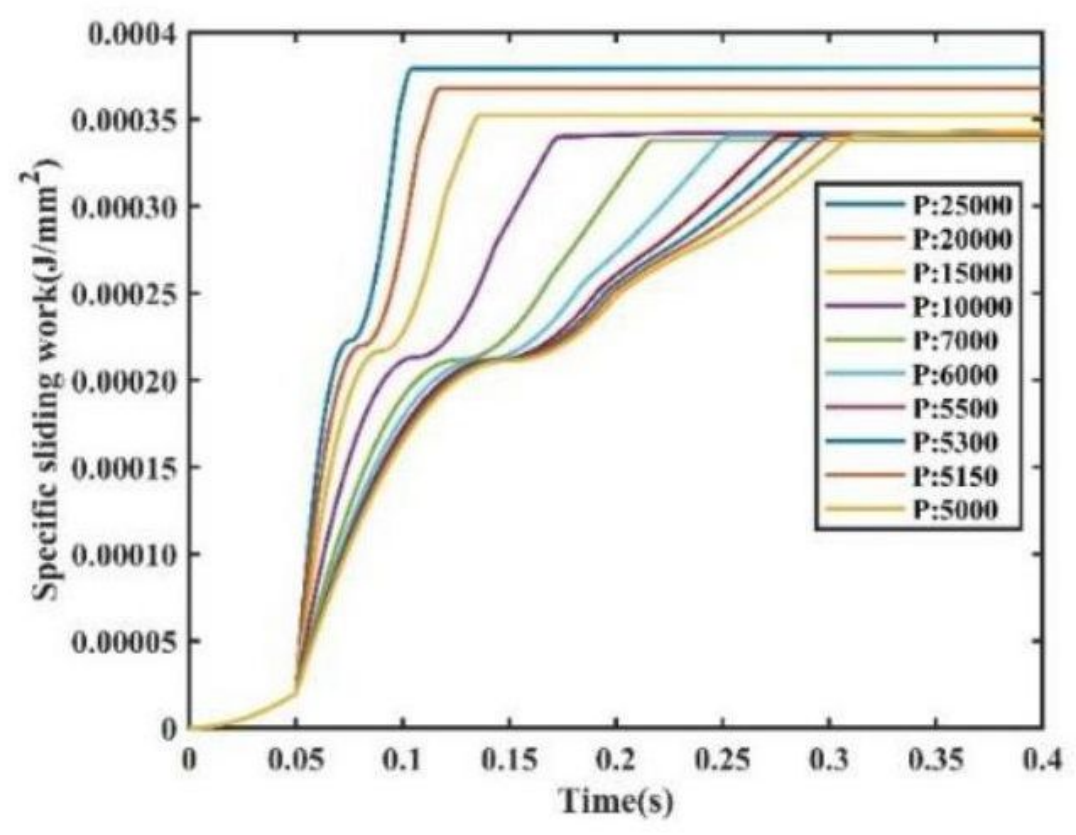

(a) Specific sliding work curves

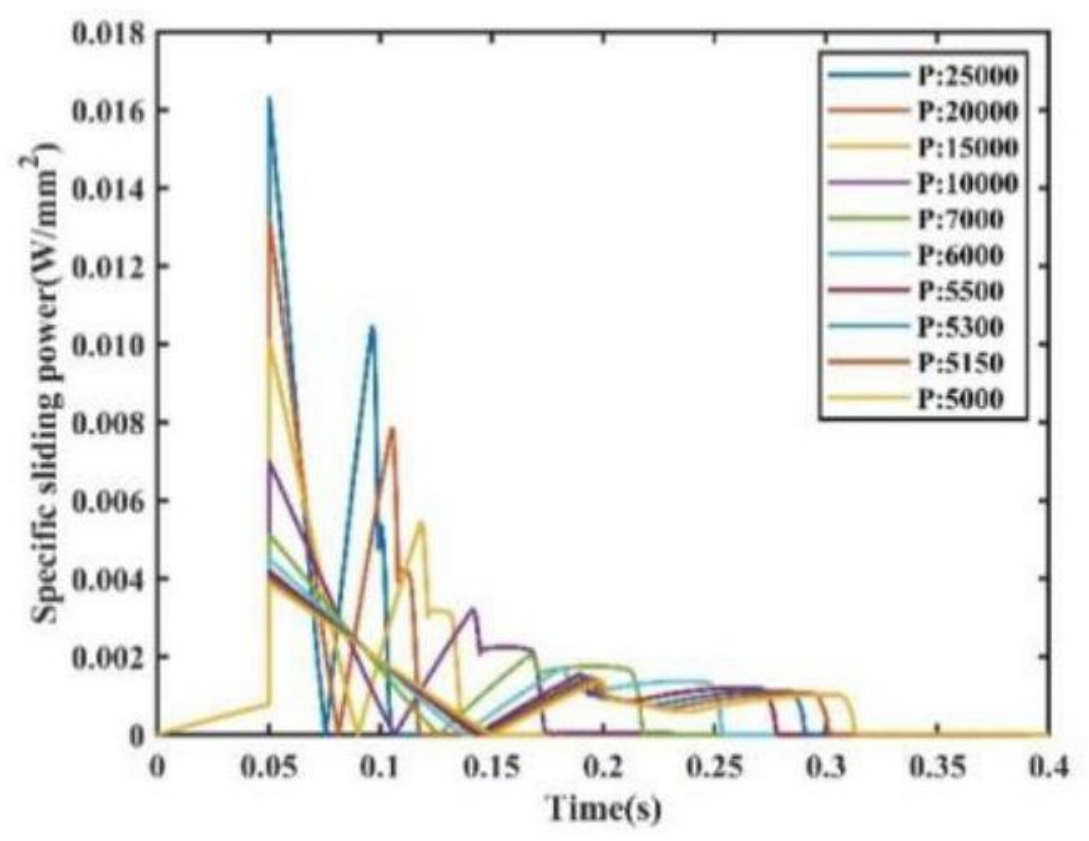

(b) Specific sliding power curves

Figure 17

Using different shifting forces to directly realize position-controlled shifting 


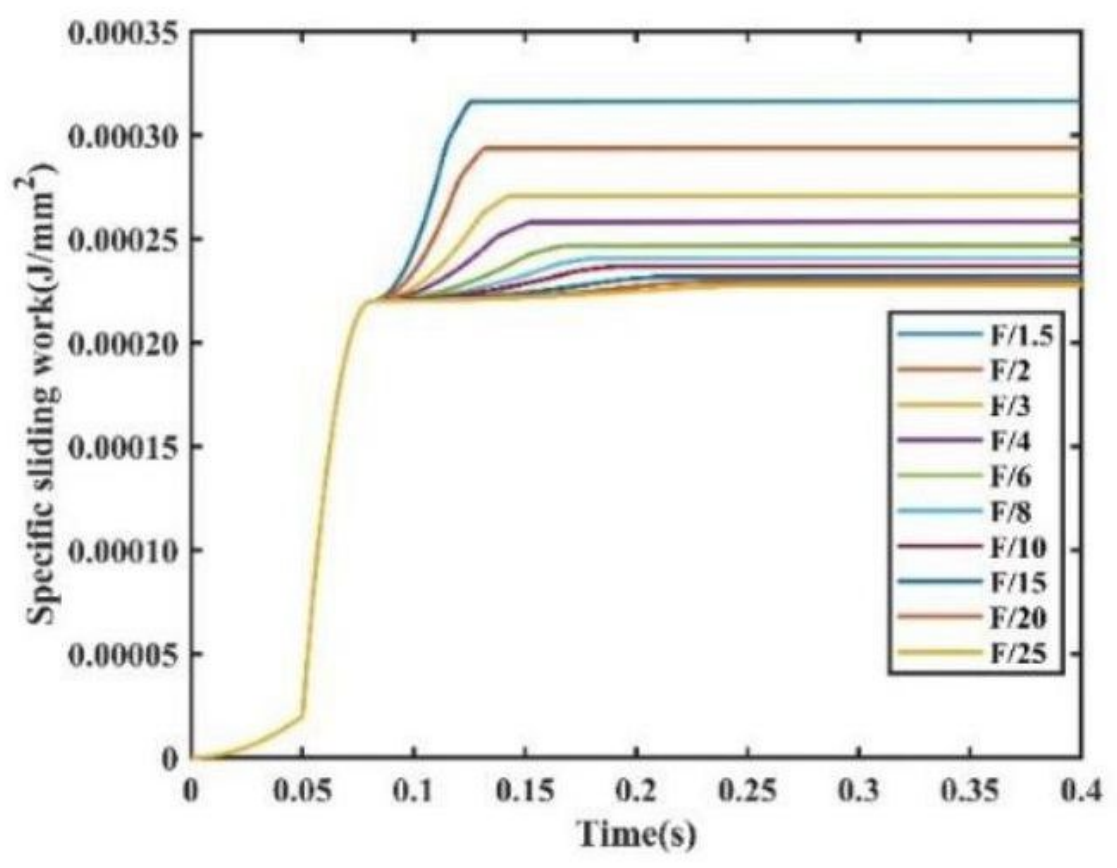

(a) Specific sliding work curves

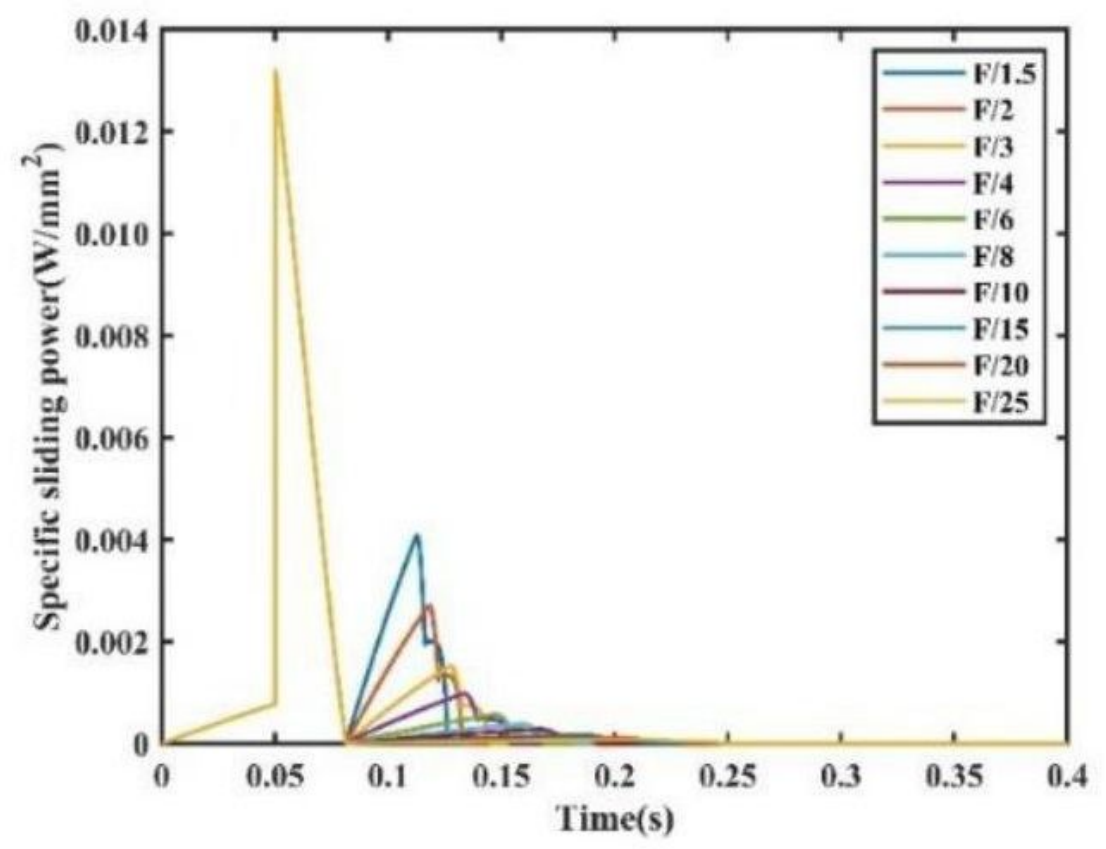

(b) Specific sliding power curves

Figure 18

Using different shifting forces after synchronization to achieve position-controlled shifting 


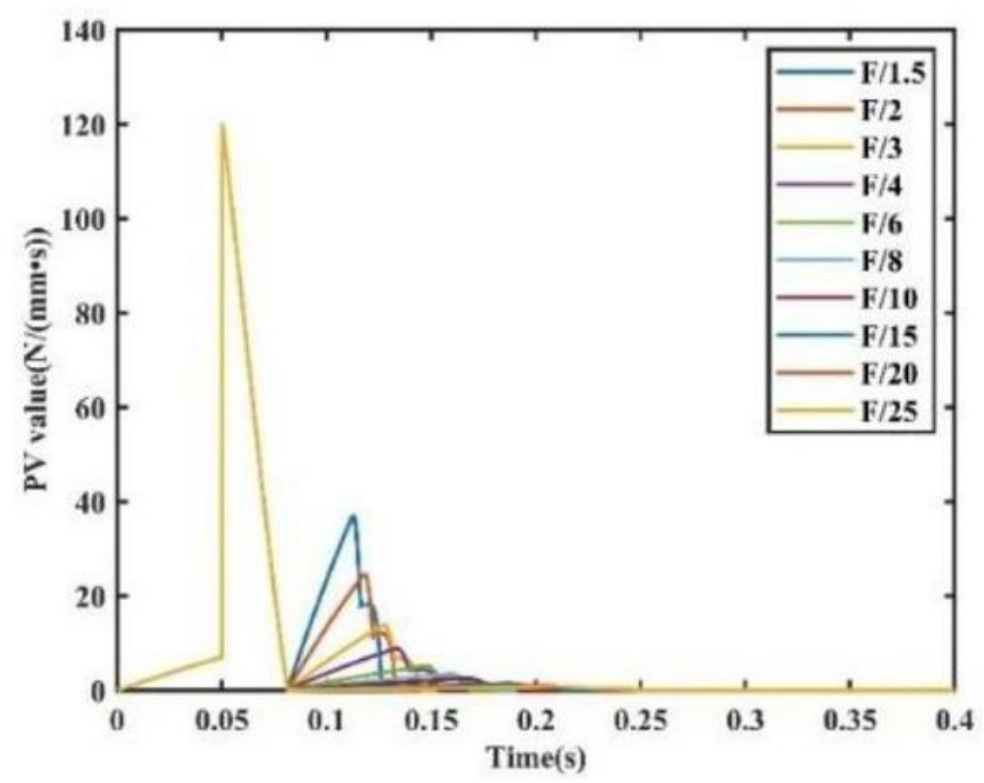

(a) PV value curves using direct position control

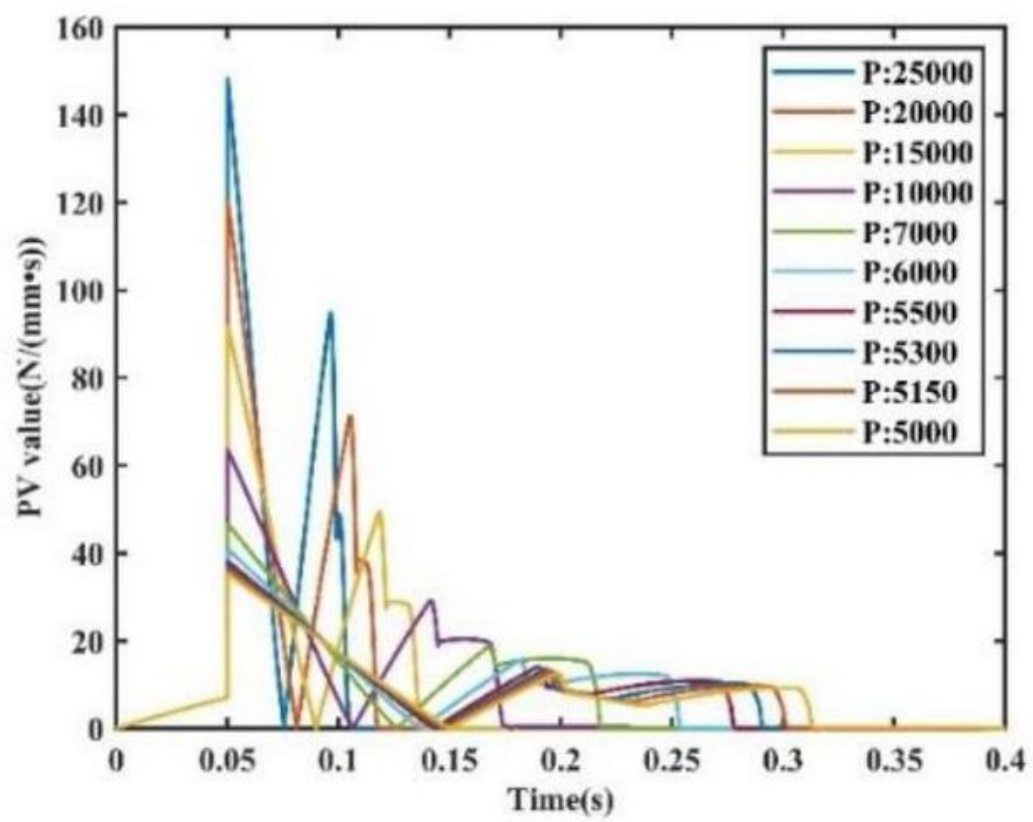

(b) PV value curves for position control with different shifting forces after synchronization

Figure 19

Synchronizer shift PV value for different shift modes 